\title{
Estructura espacial y dinámica temporal de la ocupación humana de Neuquén (Patagonia argentina) durante el Pleistoceno final-Holoceno
}

\author{
Florencia Gordón, Marien Béguelin, Diego D. Rindel, Claudia Della Negra, Adam \\ Hajduk, Romina C. Vázquez, Virginia A. Cobos, S. Ivan Perez y Valeria Bernal \\ Recibido 10 de octubre 2018. Aceptado 02 de mayo 2019
}

\begin{abstract}
RESUMEN
Se generó una base de datos conformada por 184 fechados georreferenciados procedentes de sitios arqueológicos de la provincia del Neuquén. Doce de ellos -realizados a partir de restos óseos humanos y faunísticos- son originales y algunos de ellos corresponden a áreas geográficas para las que aún no se contaba con datos. Se efectuaron análisis cuantitativos temporo-espaciales. Los resultados obtenidos sobre la base de datos cronológicos y geográficos permiten caracterizar el proceso de ocupación humana de Neuquén como temprano, mayormente continuo a lo largo del tiempo y espacialmente discontinuo. La profundidad temporal del poblamiento alcanza ca. 12.000 años calibrados AP y la ocupación del espacio se incrementó significativamente hacia el Holoceno tardío. La distribución de los sitios es discontinua y muestra concentraciones en áreas particulares cercanas a los cursos de agua permanente, mayoritariamente pericordilleranas, en tanto que en los espacios intermedios -las amplias planicies del este- no se registró una señal arqueológica marcada.
\end{abstract}

Palabras clave: Espacial; Cronología; Patagonia.

\section{ABSTRACT}

SPATIAL STRUCTURE AND TEMPORAL DYNAMICS OF THE HUMAN OCCUPATION OF NEUQUÉN (ARGENTINIAN PATAGONIA) DURING THE LATE PLEISTOCENE - HOLOCENE. A database was generated consisting of 184 georeferenced dates from archaeological sites in Neuquén Province. Twelve of these dates -taken from human and faunal bone remains- are original, and some correspond to geographical

\footnotetext{
Florencia Gordón. Consejo Nacional de Investigaciones Científicas y Técnicas (CONICET), División Antropología, Facultad de Ciencias Naturales y Museo, Universidad Nacional de La Plata. Calle 60 y 122 (1900), La Plata, Buenos Aires. E-mail: fgordon2007@gmail.com

Marien Béguelin. CONICET, Instituto de Investigación en Paleobiología y Geología, Universidad Nacional de Río Negro. Av. Roca 1242 (8332), Gral. Roca, Río Negro. E-mail: mbeguelink@gmail.com

Diego D. Rindel. CONICET, Instituto Nacional de Antropología y Pensamiento Latinoamericano. 3 de Febrero 1378 (1426), Ciudad Autónoma de Buenos Aires. E-mail: drindelarqueo@yahoo.com

Claudia Della Negra. Dirección de Patrimonio Material, Subsecretaría de Cultura, Gobierno de la Provincia de Neuquén. Vuelta de Obligado 50 (8300), Ciudad de Neuquén. E-mail: claudellanegra@hotmail.com

Adam Hajduk. Museo de la Patagonia "Francisco P. Moreno", Centro Cívico s/nº (8400), San Carlos de Bariloche, Río Negro. E-mail: ahtrapalanda@gmail.com

Romina C. Vázquez. CONICET, Instituto de Investigación en Paleobiología y Geología, Universidad Nacional de Río Negro. Av. Roca 1242 (8332), Gral. Roca, Prov. Río Negro. E-mail: rominacvazquez@gmail.com

Virginia A. Cobos. CONICET, División Antropología, Facultad de Ciencias Naturales y Museo, Universidad Nacional de La Plata. Calle 60 y 122, (1900), La Plata, Prov. Buenos Aires. E-mail: virginiacobos@gmail.com

S. Ivan Perez. CONICET, División Antropología, Facultad de Ciencias Naturales y Museo, Universidad Nacional de La Plata. Calle 60 y 122, (1900), La Plata, Prov. Buenos Aires. E-mail: ivanperezmorea@gmail.com

Valeria Bernal. CONICET, División Antropología, Facultad de Ciencias Naturales y Museo, Universidad Nacional de La Plata. Calle 60 y 122, (1900), La Plata, Prov. Buenos Aires. E-mail: bernal.valeria@gmail.com
} 
areas for which data were not previously available. Quantitative spatial-temporal analyzes were carried out. The results obtained on the basis of chronological and geographical data allow Neuquén's human occupation process to be characterized as early, mostly continuous over time, and spatially discontinuous. The temporal depth of the settlement reaches ca. 12,000 years BP calibrated, with spacial occupation increasing significantly towards the late Holocene. The spatial distribution of the sites is discontinuous and shows concentrations in particular areas near the permanent water courses, mostly near the Andes, while in the intermediate spaces -the wide plains of the east- a marked archaeological signal was not recorded.

Keywords: Spatial; Chronology; Patagonia.

\section{INTRODUCCIÓN}

La comprensión del proceso de ocupación humana del territorio de la actual provincia del Neuquén, desde su inicio en el Pleistoceno hasta momentos históricos, requiere de una caracterización detallada y conjunta de la estructura espacial y de la dinámica temporal del uso del espacio generada mediante datos geográficos y cronológicos precisos. En este sentido, los primeros fechados absolutos fueron realizados a mediados de la década de 1970 a partir de fragmentos cerámicos procedentes del Bajo del Añelo (Hajduk 1979). Desde los inicios de la década de 1990 se generó una cantidad considerable de fechados radiocarbónicos, provenientes de excavaciones de sitios en cuevas y aleros -particularmente, Cueva Haichol, Cueva Traful, Epullán y Piedra del Águila (Fernández 1988-1990; Crivelli Montero et al. 1993, 1996; Sanguinetti y Curzio 1996) - que se incrementó de manera lenta y sostenida hasta el presente (Lema et al. 2012; Barberena et al. 2015a, entre otros).

En años recientes, diferentes trabajos han comenzado a compilar los fechados absolutos disponibles procedentes de diversos sitios arqueológicos, lo que ha permitido obtener bases de datos con una cobertura espacial extensa que comprende alrededor de 60 sitios y dos centenares de dataciones (Perez et al. 2009, 2016a; Barberena et al. 2015a; Bernal et al. 2017). Barberena y colaboradores (2015a) recopilaron un conjunto de fechados radiocarbónicos calibrados de sitios arqueológicos de Neuquén y de la margen derecha del río Limay en la provincia de Río Negro que asignaron a tres áreas ecológicas diferenciadas, dispuestas longitudinalmente (i.e., estepa herbácea, estepa arbustiva y bosque andino). En el mismo sentido, Perez y colaboradores (2016a) analizaron las distribuciones de frecuencia de fechados radiocarbónicos calibrados en combinación con datos de ADN mitocondrial humano. Asimismo, Bernal y colaboradores
(2017) estudiaron, mediante el empleo de técnicas estadísticas espaciales, la estructura geográfica de un subconjunto de los sitios arqueológicos, los sitios con entierros humanos. Desde una perspectiva metodológica, estos trabajos no han explorado la influencia de sesgos de muestreo, tafonómicos (aunque algunos han aplicado un factor de corrección, como Perez et al. 2016a, b) y/o del grado diferencial del desarrollo de las investigaciones (i.e., "riqueza del excavador"), entre otros. En este sentido, resta evaluar en qué medida los patrones descritos representan la densidad diferencial de ocupación del espacio en el pasado (Surovell et al. 2009; Shennan et al. 2013; Torfing 2015).

Asimismo, las dimensiones espacial y temporal del registro arqueológico han sido analizadas de manera aislada. Los resultados obtenidos han mostrado, de forma independiente, un aumento en la frecuencia de fechados con posterioridad a los 5000-4000 años cal. AP -lo que sugiere un incremento en la densidad de ocupación poblacional humana hacia el Holoceno tardío- y una distribución de sitios arqueológicos espacialmente heterogénea a lo largo de grandes áreas definidas con criterios ecológicos (Barberena et al. 2015a), así como de sitios bioarqueológicos asociados a áreas particulares del espacio que presentan cursos de agua (Bernal et al. 2017). Sin embargo, la ocupación humana del espacio ocurre naturalmente en un contexto espacio-temporal y, en ciertos casos, puede covariar a lo largo de estas dimensiones. En este sentido, la presencia humana en una región geográfica y en un momento dado puede variar a escala local debido a cambios en las condiciones climáticas a corto y mediano plazo. Por ejemplo, las ocupaciones en el noroeste de Patagonia podrían haber variado en función de eventos de glaciación, sequía/aridez, vulcanismo (Stine 1994; D'Elia et al. 2014), cuyo efecto fue espacialmente heterogéneo. Si se considerara exclusivamente la dimensión temporal a una escala amplia -el noroeste de Patagonia-, se podría 
postular la continuidad en la ocupación aun cuando en ciertos momentos algunas áreas no estuvieron pobladas. Por esto, resulta necesario caracterizar el patrón espacio-temporal de la ocupación humana considerando de manera conjunta los fechados absolutos y las coordenadas geográficas de los sitios arqueológicos de Neuquén. El empleo de análisis espacio-temporales en un marco analítico formal permitirá evitar sesgos derivados de considerar exclusivamente una de las dimensiones -espacial o temporal-, así como modelar de manera realista los cambios en la ocupación humana.

En este trabajo se analiza de manera conjunta información cronológica y geográfica, -i.e., fechados absolutos georreferenciados- de sitios arqueológicos de la provincia del Neuquén con el fin de profundizar la comprensión del proceso de ocupación humana en la región a lo largo del Pleistoceno final-Holoceno. Para ello: (a) se obtuvieron 12 nuevos fechados radiocarbónicos realizados a partir de restos óseos humanos y faunísticos procedentes de sitios arqueológicos de la provincia, entre los cuales se incluyen algunos de áreas geográficas para las que aún no se contaba con datos; (b) se efectuaron análisis cuantitativos temporales y espaciales sobre una base de datos conformada por 184 fechados georreferenciados; y (c) se evaluaron los sesgos tafonómicos y del grado diferencial de desarrollo de las investigaciones (i.e., "riqueza" del excavador).

\section{MATERIALES $Y$ MÉTODOS}

Se generó una base de datos que contiene un total de 172 fechados radiocarbónicos georreferenciados publicados, correspondientes a sitios arqueológicos a cielo abierto, cuevas y aleros y entierros humanos de la provincia del Neuquén, noroeste de Patagonia, Argentina (Figura 1, Tabla 1, Tabla 1S). Estos fechados fueron realizados a partir de hueso humano y de fauna, carbón y cerámica mediante técnicas de radiocarbono convencional y espectrometría de masa con aceleradores (AMS), así como por termoluminiscencia. Asimismo, se determinó la edad radiocarbónica (AMS) de un conjunto de 12 muestras óseas de humanos y fauna recuperados en excavaciones de diversos sitios arqueológicos de la provincia. En este trabajo se presentan fechados para los sitios Chacra Álvarez, Hermanos Lazcano y Aquihuecó (Dpto. de Chos Malal), Chorriaca (Dpto. Loncopué), Piera (Dpto. Picunches), Cochico-Márquez (Dpto. Pehuenches) y Cueva Ruka (Dpto. Añelo) (Tabla 2). Con excepción de Aquihuecó (Della Negra y Novellino 2005; Della Negra et al. 2009) y Hermanos Lazcano (Della Negra et al. 2014), ambos ubicados en el departamento de Chos Malal, ninguno de los sitios contaba con dataciones radiocarbónicas previas. Las nuevas dataciones fueron realizadas en el Scottish Universities Environmental Research Centre (SUERC; Scotland, UK) y en el Arizona Radiocarbon AMS facility (University of Arizona). 


\begin{tabular}{|c|c|c|c|c|c|}
\hline Ref. & Sitio & Longitud & Latitud & Tipo1 & Tipo2 \\
\hline 1 & Aguada del Overo & $-70,1666$ & $-39,6666$ & Cielo abierto & Entierro \\
\hline 2 & Alero Cicuta & $-71,1713$ & $-40,6812$ & Alero & Actividades múltiples \\
\hline 3 & Alero del Dique & $-68,4886$ & $-38,999$ & Alero & Actividades múltiples \\
\hline 4 & Alero de los Álamos & $-68,4886$ & $-38,999$ & Alero & Actividades múltiples \\
\hline 5 & Alero de los Sauces & $-68,7667$ & $-39,2333$ & Alero & Actividades múltiples \\
\hline 6 & Alero Lariviere & $-71,2283$ & $-40,6021$ & Alero & Actividades múltiples \\
\hline 7 & Alero Las Mellizas & $-71,3584$ & $-40,6037$ & Alero & Actividades múltiples \\
\hline 8 & Alero Los Cipreses & $-71,3187$ & $-40,6431$ & Alero & Actividades múltiples \\
\hline 9 & Aquihueco & $-70,3765$ & $-37,0933$ & Cielo abierto & Entierro/Actividades múltiples \\
\hline 10 & Arroyo Corral 2 & $-71,0503$ & $-40,9305$ & Cueva & Actividades múltiples \\
\hline 11 & Arroyo Dulce IV & $-71,07789$ & $-37,8545$ & Cielo abierto & Actividades múltiples \\
\hline 12 & Chacra Álvarez & $-70,2674$ & $-37,3916$ & Cielo abierto & Entierro \\
\hline 13 & Chacra Bustamante & $-69,05$ & $-39,05$ & Cielo abierto & Entierro \\
\hline 14 & Chorriaca & $-70,1015$ & $-37,9381$ & Cielo abierto & Entierro \\
\hline 15 & Cochico Márquez & $-70,2213$ & $-36,4841$ & Cielo abierto & Entierro \\
\hline 16 & Collón Chico & $-68,1069$ & $-38,9667$ & Cielo abierto & Actividades múltiples \\
\hline 17 & Cueva del Choique & $-70,0046$ & $-40,0702$ & Cueva & Actividades múltiples \\
\hline 18 & Cueva Huenul & $-69,8237$ & $-36,9587$ & Cueva & Actividades múltiples \\
\hline 19 & Cueva Lagartija & $-71,2774$ & $-40,7011$ & Cueva & Actividades múltiples \\
\hline 20 & Cueva Parque-Diana & $-71,5798$ & $-40,1888$ & Cueva & Actividades múltiples \\
\hline 21 & Cueva Ruka & $-68,8904$ & $-37,7568$ & Cueva & Actividades múltiples \\
\hline 22 & Cueva Traful & $-71,13$ & $-40,7304$ & Cueva & Entierro/Actividades múltiples \\
\hline 23 & Cuyin Manzano & $-71,1873$ & $-40,7631$ & Cueva & Actividades múltiples \\
\hline 24 & Epullan Chica & $-70,1841$ & $-40,3904$ & Cueva & Actividades múltiples \\
\hline 25 & Epullan Grande & $-70,1841$ & $-40,3904$ & Cueva & Entierro/Actividades múltiples \\
\hline 26 & Gubevi & $-70,6697$ & $-37,1773$ & Cielo abierto & Entierro \\
\hline 27 & Haichol & $-70,6714$ & $-38,5858$ & Cueva & Entierro/Actividades múltiples \\
\hline 28 & Hermanos Lazcano & $-70,458854$ & $-37,261235$ & Cielo abierto & Entierro \\
\hline 29 & Lago Meliquina & $-71,3169$ & $-40,3325$ & Cielo abierto & Actividades múltiples \\
\hline 30 & Laguna Montículo I & $-70,4823$ & $-38,8584$ & Cielo abierto & Actividades múltiples \\
\hline 31 & La Oquedad & $-71,2471$ & $-40,6641$ & Alero & Actividades múltiples \\
\hline 32 & Las Cachañas & $-71,5379$ & $-40,2333$ & Cielo abierto & Actividades múltiples \\
\hline 33 & Llamuco 1 & $-70,0354$ & $-38,7556$ & Cielo abierto & Actividades múltiples \\
\hline 34 & Loma de la Lata & $-68,6833$ & $-38,4167$ & Cielo abierto & Entierro \\
\hline 35 & Malal Huaca & $-71,0726$ & $-40,6754$ & Alero & Actividades múltiples \\
\hline 36 & Alero IV de Tromen & $-70,444$ & $-38,8605$ & Alero & Entierro \\
\hline 37 & Mata Molle & $-70,7167$ & $-40,0874$ & Cielo abierto & Actividades múltiples \\
\hline 38 & Médano Chato & $-68,9243$ & $-38,1862$ & Cielo abierto & Actividades múltiples \\
\hline 39 & Médanos del Gigante & $-68,9$ & $-39,4$ & Cielo abierto & Entierro \\
\hline 40 & Michacheo & $-70,1716$ & $-38,8939$ & Cielo abierto & Actividades múltiples \\
\hline 41 & Montículo Angostura & $-71,26$ & $-38,8638$ & Cielo abierto & Entierro \\
\hline 42 & Moro 1 & $-68,812$ & $-39,253$ & Cueva & Actividades múltiples \\
\hline 43 & Piedra del Águila 11 & $-70,0153$ & $-40,0378$ & Cueva & Actividades múltiples \\
\hline 44 & Piera & $-69,9832$ & $-38,7033$ & Cielo abierto & Entierro \\
\hline 45 & Planicie del Gigante & $-68,9$ & $-39,4$ & Cielo abierto & Actividades múltiples \\
\hline 46 & Pocaullo & $-71,3166$ & $-40,1333$ & Cielo abierto & Entierro \\
\hline 47 & Puerto Tranquilo & $-71,5407$ & $-40,8877$ & Alero & Actividades múltiples \\
\hline 48 & Retamal 1 & $-68,71666667$ & $-38,98333333$ & Cielo abierto & Entierro \\
\hline 49 & Rincón Chico-2 & $-70,0371$ & $-40,2038$ & Alero & Actividades múltiples \\
\hline 50 & Sitio Grande & $-69,25$ & $-39,6116$ & Cielo abierto & Entierro \\
\hline 51 & Sitio Rosita Quevedo & $-70,4039$ & $-37,1582$ & Alero & Actividades múltiples \\
\hline 52 & Traful III & $-71,13$ & $-40,7304$ & Cueva & Actividades múltiples \\
\hline 53 & Truquico & $-70,6806$ & $-37,1757$ & Cueva & Actividades múltiples \\
\hline 54 & Villa Traful & $-71,4355$ & $-40,6631$ & Cielo abierto & Actividades múltiples \\
\hline
\end{tabular}

Tabla 1. Sitios estudiados en este trabajo, considerando su ubicación geográfica y el tipo de cada uno de ellos. Las referencias corresponden a los números consignados en la figura 1. 


\begin{tabular}{|l|c|c|l|l|l|}
\hline \multicolumn{1}{|c|}{ Sitio } & Sigla & Fechado radiocarbónico & \multicolumn{1}{|c|}{ Código laboratorio } & Tipo de sitio & Material fechado \\
\hline Chacra Álvarez & ChAl-Ind1 & $330 \pm 32$ & SUERC-77690 (GU46600) & Cielo abierto & Hueso humano \\
\hline Chorriaca & Chor & $1152 \pm 33$ & SUERC-77691 (GU46601) & Alero & Hueso humano \\
\hline Piera & Piera & $229 \pm 32$ & SUERC-77692 (GU46602) & Alero & Hueso humano \\
\hline Cochico-Márquez & Co-Mar & $2449 \pm 34$ & SUERC-77693 (GU46603) & Cielo abierto & Hueso humano \\
\hline Hermanos Lazcano & HLazC4I1 & $4016 \pm 33$ & SUERC-77697 (GU46604) & Cielo abierto & Hueso humano \\
\hline Cueva Ruka & Ruca N7 CB & $96 \pm 32$ & SUERC-77698 (GU46605) & Cueva & Hueso guanaco \\
\hline Cueva Ruka & Ruca N3 CB & $5147 \pm 37$ & SUERC-77699 (GU46606) & Cueva & Hueso guanaco \\
\hline Aquihuecó & Aqcol43 & $4199 \pm 35$ & SUERC-77700 (GU46607) & Cielo abierto & Hueso humano \\
\hline Aquihuecó & AQ1-27 & $4280 \pm 59$ & AA101834 & Cielo abierto & Hueso humano \\
\hline Aquihuecó & AQ1-33 & $4045 \pm 66$ & AA101835 & Cielo abierto & Hueso humano \\
\hline Aquihuecó & AQ1-34 & $4304 \pm 59$ & AA101836 & Cielo abierto & Hueso humano \\
\hline Aquihuecó & AQ1-40 & $4165 \pm 64$ & AA101837 & Cielo abierto & Hueso humano \\
\hline
\end{tabular}

con el fin de describir los cambios temporales en la intensidad de ocupación h u m a n a considerando diferentes tipos de sitios (cueva + aleros Tabla 2. Fechados radiocarbónicos originales incluidos en este trabajo.

versus cielo abierto) $y$

El total de los 184 fechados (172 publicados previamente y 12 originales de este trabajo) fueron analizados con el fin de comprender los cambios espacio-temporales en la ocupación humana en la provincia del Neuquén. En primer lugar, los fechados fueron calibrados en el package rcarbon para el programa R (Crema et al. 2017), empleando la curva para el hemisferio sur 'shcal13'. Las calibraciones fueron corroboradas mediante el programa Calib 7.0.4 utilizando la misma curva (Stuiver y Reimer 1993). Los valores obtenidos por ambos programas fueron muy similares, ya que no difieren en más de un año. En segundo lugar, se agruparon y promediaron los fechados de un mismo sitio arqueológico que estaban incluidos en intervalos menores a 200 y 100 años radiocarbónicos con el fin de evitar el sobremuestreo del mismo evento de ocupación (Timpson et al. 2014; Perez et al. 2016a y b). Debido a que ambos intervalos arrojaron resultados similares, se empleó el intervalo menor en los siguientes análisis; se promediaron 22 conjuntos de fechados (i.e., bins) y se excluyeron 28 datos en la base final. Asimismo, se eliminaron de la base de datos los fechados menores a 500 años AP (n = 14) (ver detalles en Tabla 1S), ya que el muestreo en el período reciente es muy bajo debido a decisiones de los investigadores, quienes generalmente utilizan indicadores cronológicos relativos (e.g., presencia de metales, etc.). El conjunto final de datos empleado en los siguientes análisis incluye 142 fechados radiocarbónicos con un rango entre ca. 500 y 12.500 años cal. AP.

A partir de los 142 fechados radiocarbónicos calibrados se efectuó un análisis no-paramétrico de permutación por pares para la Distribución de Probabilidad Sumada (rPerm-SPD, por sus siglas en inglés) (Timpson et al. 2014; Crema et al. 2016), regiones del territorio de la provincia (bosques, estepa arbustiva y estepa herbácea sensu Barberena et al. 2015a), empleando el paquete rcarbon para el programa R (Crema et al. 2017). Este análisis fue desarrollado recientemente por Shennan y colaboradores (Shennan et al. 2013; Timpson et al. 2014; Crema et al. 2016) y focaliza en los cambios relativos en la frecuencia de fechados radiocarbónicos en el tiempo y en la comparación de diferentes conjuntos de datos. En este trabajo, la comparación de diferentes conjuntos de datos permitió evaluar/ explorar los sesgos tafonómicos y del grado diferencial de desarrollo de investigaciones (i.e., "riqueza" del excavador). Las SPDs fueron suavizadas mediante el cálculo de un promedio móvil sobre un intervalo de 200 años, tal como lo sugieren trabajos previos (Timpson et al. 2014; Crema et al. 2016).

La estimación de la distribución espacial de fechados absolutos en diferentes momentos en el tiempo requiere de la definición de intervalos temporales (Crema et al. 2017; Perez et al. 2016a y b). Considerando la cantidad -relativamente bajade fechados disponibles para la construcción de la curva de Probabilidad Sumada y que la curva presenta agrupamientos de fechados bien definidos (ver Resultados), se establecieron tres intervalos temporales o períodos de 3000 y, el más temprano, de 2500 años. Para estimar las diferencias geográficas en la frecuencia de sitios, y por lo tanto en el patrón de ocupación humana, para cada período se generó un mapa suavizado empleando el método de densidad espacial de Kernel. Se graficó la distribución de sitios para los dos primeros períodos -i.e., 12.000-9500 y 9500-6500- y se efectuó un análisis de densidad de sitios empleando la función mapa de calor (Heatmap) para los dos períodos restantes. La estimación del mapa de densidad, o 
mapa de calor, de los sitios fue efectuada usando los siguientes parámetros del algoritmo de Kernel: radio = 1 grado; forma del Kernel = cuártico; $y$ valor de escalamiento $=0$. Asimismo, se calculó la correspondencia entre la frecuencia de sitios en grillas espaciales de $0,5 \times 0,5$ grados y las áreas ecológicas, hipotetizadas como relevantes para explicar la variación en los sitios por Barberena et al. (2015a), mediante el método de Chi-2 con corrección para datos con frecuencias menores a cinco. Los análisis espaciales fueron efectuados en el programa QGIS 3.4.0.

\section{RESULTADOS}

De los 54 sitios (Figura 1) compilados en la base de datos, 26 corresponden a sitios a cielo abierto, 15 a cuevas y 13 a aleros; en tanto que, del total de sitios, 19 presentan entierros humanos y 35 pueden ser asignados a actividades múltiples. Es de destacar que en la base final de fechados $(N=142)$, los sitios en cueva y alero -distribuidos de manera similar en los diferentes sectores de la provincia- son los que presentan una mayor frecuencia de fechados $(\mathrm{n}=108)$, en relación con los sitios a cielo abierto $(n=34)$. En este mismo sentido, todas las dataciones calibradas (cal.) que superan los 5000 años AP -con la excepción de un fechado del sitio Aquihuecó de ca. 9000 años cal. AP- corresponden a sitios en cuevas. El registro bioarqueológico mostró una gran profundidad temporal y una frecuencia mayor de sitios datados en el Holoceno tardío final. Asimismo, los datos de sitios de actividades múltiples muestran diferencias en la frecuencia en distintos momentos y espacios geográficos; es destacable que la cantidad de datos disponibles es menor en el norte de la provincia y que la mayor parte de los fechados tempranos se realizaron sobre materiales hallados en cuevas del sur de esta (Tabla 1S).

La Distribución de Probabilidad Sumada de los fechados calibrados a lo largo del tiempo muestra un patrón caracterizado por una frecuencia relativamente baja y continua, con valores de alrededor de 0.05 de probabilidad sumada, entre 6500 y 12.000 años cal. AP, aunque con un hiato de ca. 500 años -entre 9500 y 10.000 años cal. AP- sin evidencia de ocupación humana en la región (Figura 2). Después del 6500 AP y hasta ca. 3500 años cal. AP, la intensidad de ocupación humana aumenta en Neuquén a una tasa moderada, ya que presenta un decrecimiento relativo entre ca. 3000 y 4000 años cal. AP. Entre el contacto hispano-indígena y los 3000 años cal. AP, la cantidad de fechados aumentó a una tasa mucho mayor que la registrada hasta ese momento, y alcanza valores de aproximadamente 0.10 de probabilidad sumada (Figura 2). Es de destacar que cuando consideramos los fechados en cuevas y aleros de manera separada de los sitios a cielo abierto, el aumento observado en el Holoceno tardío es pronunciado, aunque en menor medida (Figura 2). En este sentido, la ausencia de fechados para sitios a cielo abierto se relacionaría con factores tafonómicos (Surovell et al. 2009).

La Figura 3 muestra que la ocupación humana no fue homogénea a lo largo del espacio. En este sentido, se observa una mayor frecuencia de fechados en el sur, principalmente en la cuenca sur del río Limay, así como en el extremo norte de la cordillera y en la cuenca del río Barrancas. También se observan áreas que presentan vacíos de información, en especial el sector norte de la provincia (deptos. de Añelo, Pehuenches, Chos Malal, Minas, Ñorquín, Loncopué y Picunches), así como en los espacios intermedios alejados de los cursos de agua permanentes. Es importante notar que la alta intensidad de ocupación inferida para el centro-oeste de la provincia (Figura 3) se vincula con la alta frecuencia de fechados efectuados en un único sitio, Cueva de Haichol, por lo que debe ser considerada con precaución. El conjunto final de datos analizado para este sitio fue solo de 23 de los 44 fechados disponibles. Si bien se excluyeron 21 -aquellos que difieren en menos de 100 años de otros fechados del sitio-, el número continúa siendo relativamente elevado, en particular para el período 3500/6500 años cal. AP, en comparación con los obtenidos para otras cuevas -que ronda los diez fechados-. Esto sugiere la existencia de un sesgo de "riqueza" del excavador.

Al considerar los cambios temporales en la ocupación del espacio, se observa que durante el primer período -9500/12.500 años cal. AP-, solo se ocupa el espacio en el norte y sur de Neuquén, sobre las cuencas de los ríos Barrancas y Limay (Figura 4). Entre 6500/9500 años cal. AP, el patrón espacial resulta similar al del periodo anterior, aunque se observan fechados en el centro-oeste de la provincia, correspondientes al sitio Cueva de Haichol. La ocupación comienza a ser más intensa en estas áreas en el período que abarca entre 3500/6500 años cal. AP, y se registran las primeras evidencias de ocupación humana, aunque escasas, en el este de la 

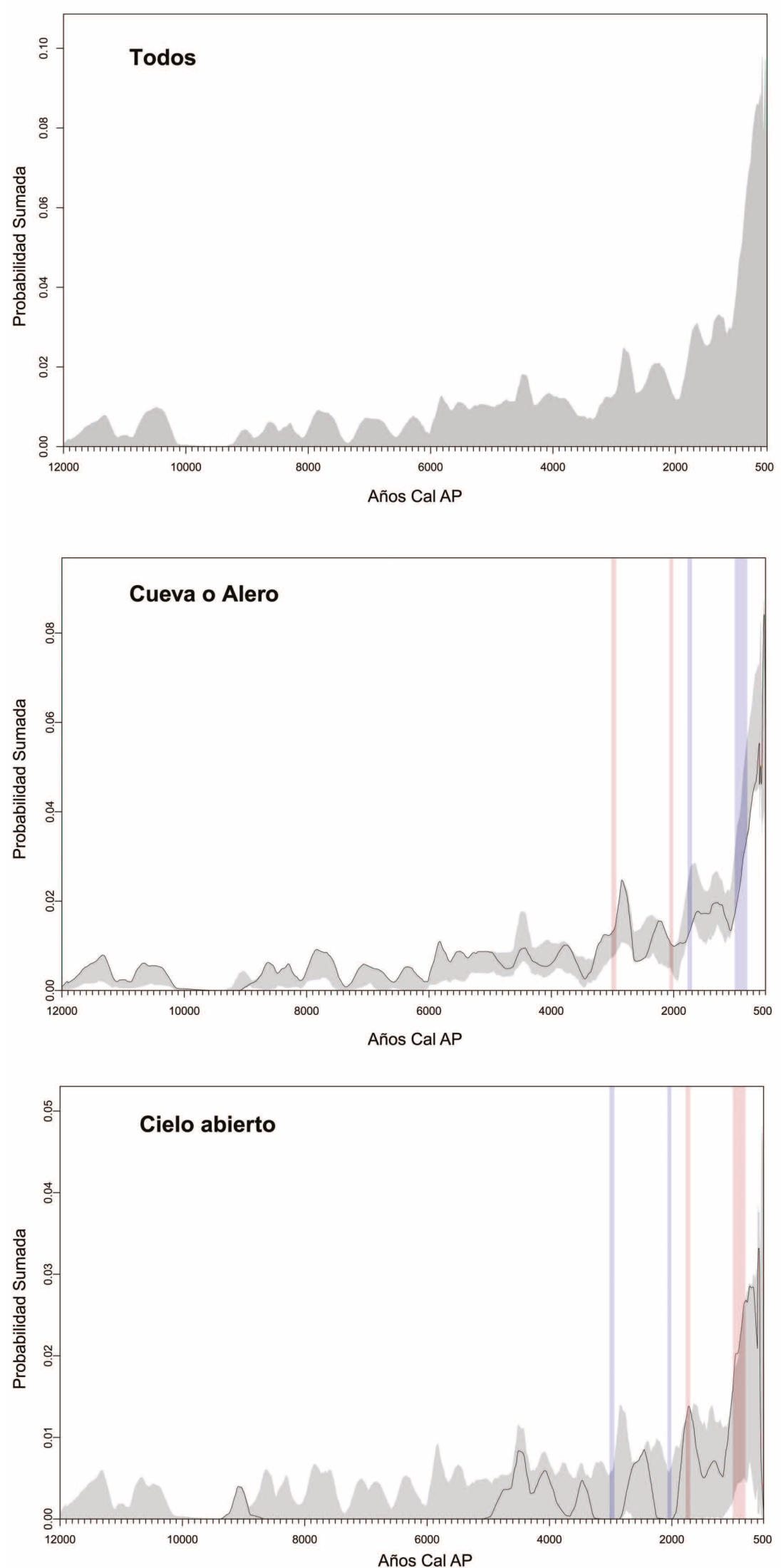

Figura 2. Distribución de Probabilidad Sumada de todos los fechados radiocarbónicos considerados y resultado del análisis no-paramétrico de permutación por pares comparando la Distribución de Probabilidad Sumada de sitios de Cueva + Aleros versus Cielo Abierto. provincia (Figura 4). En el último período estudiado, entre $500 / 3500$ años cal. AP, es en el que se observa la mayor intensidad de ocupación en las diferentes áreas, aunque siempre es mayor en la cordillera de los Andes, principalmente en el sur de Neuquén (Figura 4). Es de destacar que en el sector sur de la provincia se observa un aumento en la intensidad de ocupación del espacio. En este sector se encuentran representadas las tres áreas ecológicas consideradas en trabajos previos (Figura 1), lo que sugiere una escasa correspondencia entre un aumento en la densidad de ocupación y el poblamiento de áreas ecológicas particulares. En este sentido, la prueba de la correspondencia entre la frecuencia de fechados y las áreas ecológicas mostró valores no significativos $(\mathrm{Chi}-2=$ 19.18, $\mathrm{P}=0.634$ ).

\section{DISCUSIÓN}

Los resultados obtenidos en este trabajo muestran un aumento en la cantidad de fechados con posterioridad a 6500 años cal. AP, y un incremento significativo después del 3500 cal. AP. En líneas generales, estos resultados concuerdan con lo descrito por trabajos previos para el noroeste de Patagonia -una región más amplia que la estudiada aquí-, lo que muestra la existencia de un aumento significativo en la frecuencia de la ocupación humana hacia el Holoceno tardío. Sin embargo, se pueden observar algunas diferencias en relación con los patrones descriptos previamente 


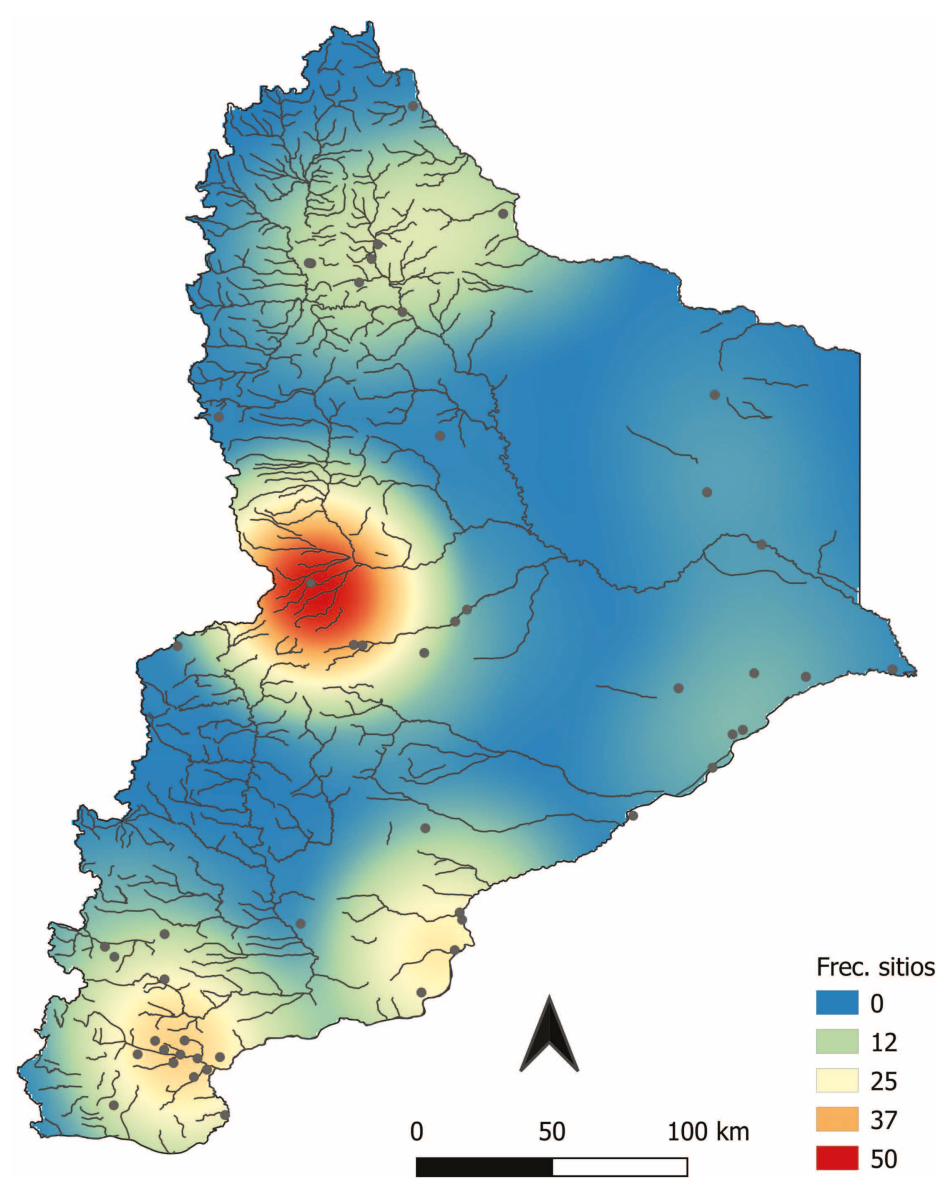

Figura 3. Distribución geográfica de la densidad de todos los fechados radiocarbónicos a partir del método espacial de Kernel.

(Barberena et al. 2015a; Perez et al. 2016a). En particular, estos estudios señalaron -considerando exclusivamente la variable temporal- un aumento importante en la frecuencia de sitios con posterioridad a los 4000-5000 años cal. AP y una mayor frecuencia de sitios después del 2000 AP (Barberena et al. 2015a). Asimismo, los análisis moleculares y la distribución de fechados corregidos por sesgos tafonómicos señalan que el inicio del aumento demográfico en el noroeste de Patagonia probablemente ocurrió algunos milenios antes que lo sugerido, ca. 7500 años cal. AP, pero el incremento marcado se habría dado después del 4000-5000 AP (Perez et al. 2016a; Gordón et al. 2019).

Es importante notar que la mayor parte de los 12 fechados obtenidos aquí corresponde a contextos del Holoceno medio-tardío inicial, un momento para el que se ha propuesto, incluso, la existencia de un hiato ocupacional (Barberena et al. 2015b). En este sentido, la comparación entre los cambios en la densidad de ocupación a lo largo del territorio neuquino (Figura 2) y los observados para cada una de las áreas ecológicas (Figura 1S; Barberena et al. 2015a) sugiere que el único hiato recurrente a nivel regional es el observado entre 9500 y 10.000 años cal. AP, para el que no hay evidencia de ocupación humana en ninguna de las áreas ecológicas consideradas. Asimismo, la continuidad en la ocupación humana observada a partir de la suma de probabilidades de fechados radiocarbónicos muestra concordancia con la información paleodemográfica obtenida a partir de análisis moleculares (Perez et al. 2016a). Como fuera mencionado en trabajos previos, es posible que el hiato del Holoceno medio-tardío inicial observado en algunas áreas ecológicas constituya un efecto de condiciones de muestreo deficiente o la existencia de procesos locales de despoblamiento (Perez et al. 2016a).

En este sentido, si bien la construcción de una base de datos de fechados radiocarbónicos y una Curva de Probabilidad Sumada a partir de ellos es un primer paso para entender la dinámica de una población humana en el pasado, esta no puede ser tomada acríticamente como un reflejo directo de fluctuaciones poblacionales reales (Surovell et al. 2009; Torfing 2015). Nuestros resultados sugieren que el patrón espaciotemporal detectado puede estar influenciado por sesgos tafonómicos, de muestreo y/o del excavador. En este contexto, es importante señalar que, cuando se excluyen sitios de algún tipo que pueden presentar valores altos de sesgos tafonómicos, como los ubicados a cielo abierto, el patrón general de cambio en la densidad de ocupación humana no se modifica significativamente, aunque los valores relativos son menores para los últimos 2000 años (Figura 2). De esta forma, consideramos que una vía importante para identificar y ponderar la existencia de sesgos es la comparación entre diferentes tipos de sitios (Surovell et al. 2009; Torfing 2015), en este caso, entre cuevas y aleros -con mejores condiciones de preservación- y sitios a cielo abierto.

Por otra parte, ni siquiera los sitios a cielo abierto pueden ser considerados como potencialmente similares en cuanto a la probabilidad de preservación. La comparación entre sitios a cielo abierto de actividades múltiples con el registro de entierros humanos permite ilustrar este punto. El registro bioarqueológico, en la mayoría de los casos, es el resultado del entierro intencional de restos humanos y, por lo tanto, está sujeto a 
procesos de formación particulares (Guichón et al. 2000; Martin et al. 2004), mientras que los sitios a cielo abierto son producto de una dinámica natural de enterramiento de los materiales. Por otra parte, los hallazgos de sitios de entierro son producto, frecuentemente, de denuncias realizadas por pobladores a las autoridades locales o provinciales antes que de prospecciones en el terreno $y$, por lo tanto, no reflejan en todos los casos el interés de

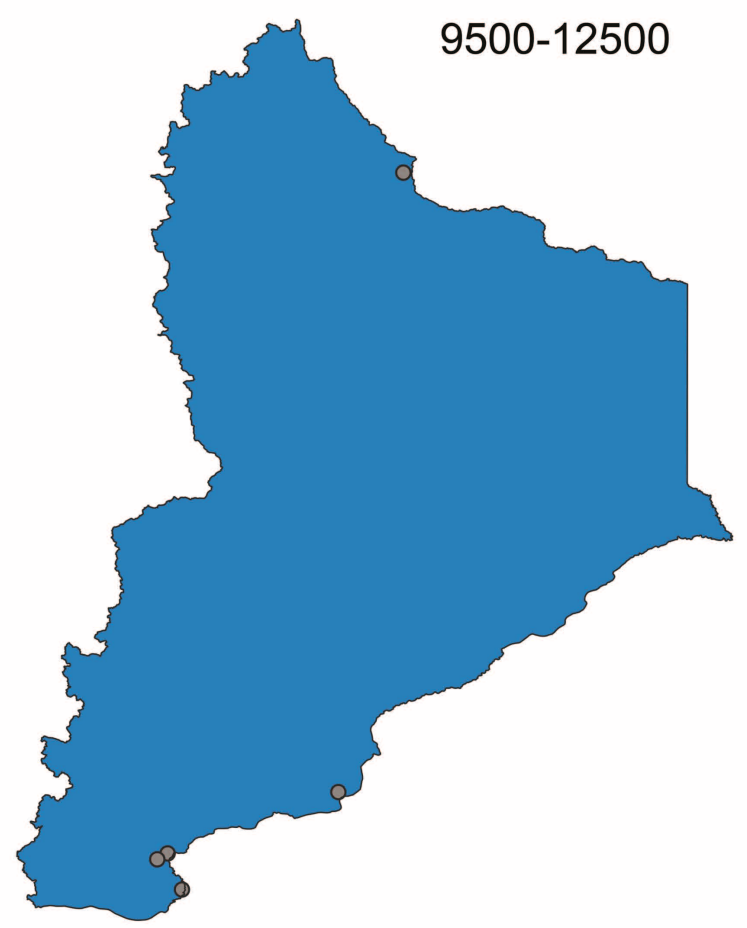

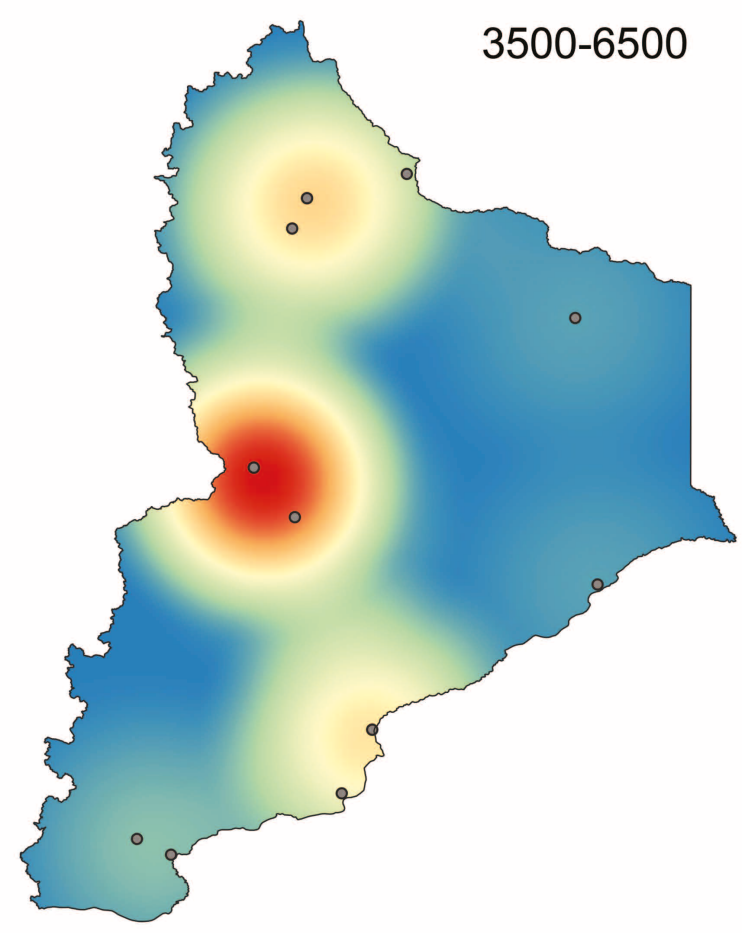

los investigadores en un momento o región en particular. En el caso de Neuquén, existen diferencias marcadas en la frecuencia entre ambos tipos de sitios - de entierro y de actividades múltiples- al considerar los sectores noroeste y suroeste de la provincia (Bernal et al. 2017). Mientras que en el noroeste existe una mayor frecuencia relativa de fechados para entierros humanos, en el suroeste predominan las dataciones para sitios del segundo
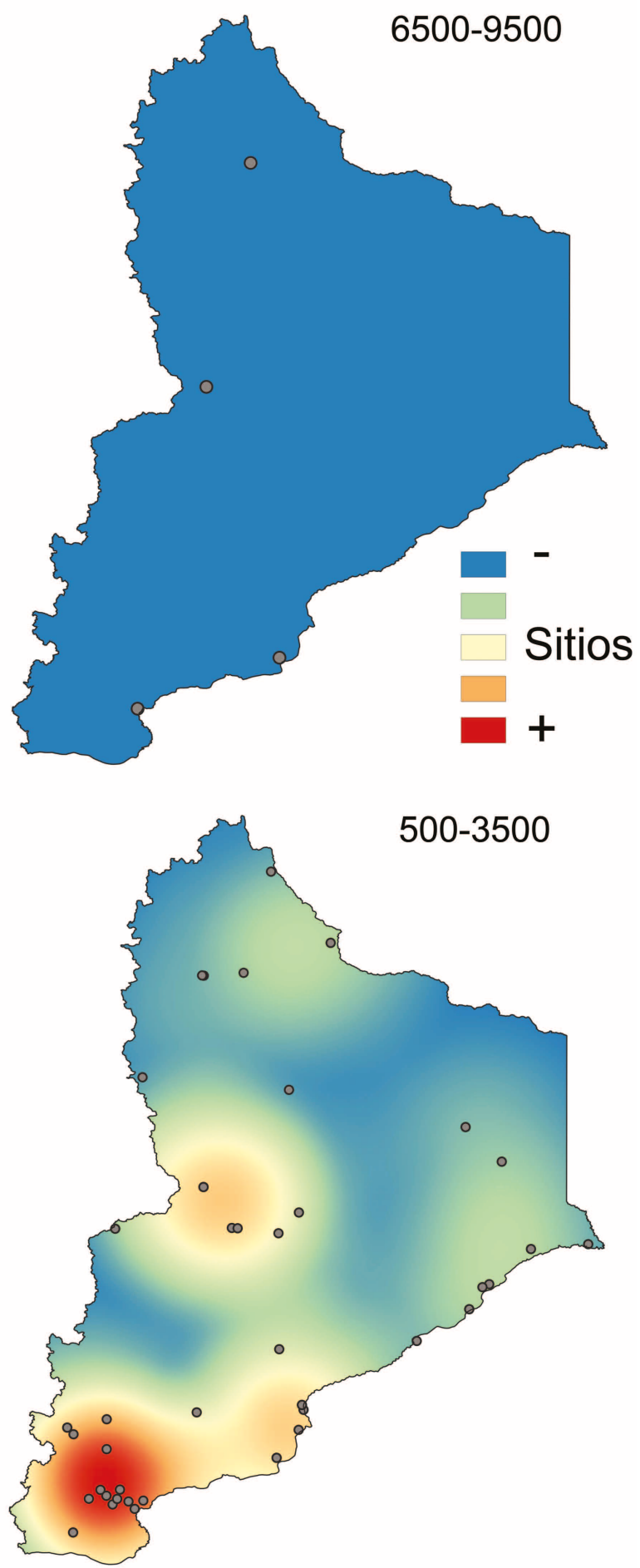

Figura 4. Distribución geográfica de la densidad de fechados radiocarbónicos por períodos a partir del método espacial de Kernel. 
tipo. En este mismo sentido, es posible discutir los nuevos fechados radiocarbónicos presentados en este trabajo. Estos muestran una frecuencia mayor en la región de montaña ubicada al oeste en relación con las zonas bajas del este de la provincia, lo que sugiere nuevamente un patrón diferencial en la ocupación humana a lo largo del Holoceno.

Bernal y colaboradores (2017) señalaron que la distribución de los sitios de entierros humanos en el espacio presenta un patrón no-aleatorio y heterogéneo, ya que exhibe una tendencia al agrupamiento de sitios en sectores particulares del espacio y que la mayor parte de los detectados corresponden al Holoceno tardío en un rango temporal que se extiende entre los 4600 años cal. AP y momentos históricos. Es de remarcar que Bernal y colaboradores (2017) también mostraron que la distribución espacial de los sitios bioarqueológicos se asocia fuertemente con los cursos de agua permanentes, como también se puede observar en la Figura 3 para todos los tipos de sitios en la región. En este sentido, la mayor frecuencia de ocupación humana en el oeste de la provincia se observa tanto con base en los sitios bioarqueológicos como para los arqueológicos en general, lo cual podría indicar cambios representativos de aquellos ocurridos en el pasado, aun cuando se incluyen posibles sesgos en el muestreo.

En cuanto a los sesgos de autor, Neuquén presenta un caso destacable, que es la alta frecuencia de fechados efectuados en Cueva de Haichol. Con el objetivo de ponderar este sesgo, se excluyeron del conjunto final de datos analizados 21 fechados del sitio, los cuales no diferían en más de 100 años de otras dataciones del mismo sitio. En este sentido, el número final de fechados de Cueva de Haichol empleados en la construcción de la curva de probabilidad sumada $(N=23)$ continúa siendo relativamente elevado, en particular para el período 3500/6500 años cal. AP, aunque se acerca al observado para otras cuevas en Neuquén $(\mathrm{N} \approx 10)$. Por este motivo, tal como sugiere Torfing (2015), es fundamental mantener una postura crítica en el análisis de las bases de fechados. En este caso particular, es importante señalar que la alta intensidad de ocupación inferida para el Holoceno medio en el centro-oeste de la provincia, representada en la Figura 4, debe ser considerada con precaución.

Los análisis de los cambios espacio-temporales del registro arqueológico de Neuquén realizados en este trabajo son de particular importancia para comprender el proceso de ocupación humana de la región. Luego de haber considerado los potenciales sesgos de la base de datos, ¿qué otros factores podrían explicar los cambios en la densidad de ocupación humana aquí observada? Los resultados obtenidos muestran que el poblamiento inicial y los cambios en el patrón de ocupación no ocurrieron de manera homogénea a lo largo de la provincia del Neuquén. Si bien estudios previos sugirieron que los fechados de la región se distribuyen heterogéneamente a lo largo de las áreas ecológicas -con un registro continuo desde 9000 años cal. AP en la estepa herbácea, y uno discontinuo en el bosque andino que tiende a regularizarse después del 4000 AP (Borrero 2005; Barberena et al. 2015a)-, nuestros resultados indican un patrón más complejo, con un aumento en la densidad de ocupación en el sur de Neuquén en el período 500-3500 años cal. AP que abarca las tres áreas ecológicas (Figura 4 y Figura 1S). Un factor común a las tres áreas representadas en el sur de Neuquén es la elevada capacidad de carga (Barberena 2013). Probablemente, el patrón detectado se relacione con procesos a escala local. En particular, es probable que la presencia de agua y la posibilidad de acceder a recursos de ambientes diferentes de manera eficiente en un área relativamente circunscripta (Crivelli Montero et al. 1993), en contraposición con las áreas de baja capacidad de carga, sea parte de la explicación para este crecimiento.

De una manera más general, Bernal y colaboradores (2017) señalan que la distribución espacial de los sitios en Neuquén podría ser el producto de procesos culturales y naturales que influyen sobre la localización de las actividades humanas, considerando variables relevantes tales como proximidad al agua, acceso a rutas, disponibilidad de recursos para la caza y recolección (Littleton 2007; Littleton y Allen 2007). Los análisis sobre el registro bioarqueológico indicaron que la proximidad a los cursos de agua permanente, así como la capacidad de carga del ambiente -en menor medida- fueron las variables relevantes para explicar la distribución de sitios de entierros humanos en Neuquén. En este mismo sentido, en nuestro trabajo se observó que, en los primeros períodos estudiados, la ocupación se registró consistentemente en las cercanías de los grandes cursos de agua permanente, ríos Limay, Barrancas y Neuquén, y se localizó en las áreas con mayor capacidad de carga, mayoritariamente pericordilleranas (Hajduk et al. 2007; Barberena 
et al. 2017; Bernal et al. 2017). Dado que, como fuera señalado, no parece existir una disponibilidad diferencial de cuevas y aleros en los diferentes sectores de la provincia, la distribución de fechados observada podría reflejar diferencias ambientales, fundamentalmente en la disponibilidad de agua, entre sectores cordilleranos y pericordilleranos y espacios esteparios áridos.

Es importante remarcar que gran parte del área este de Neuquén -que se encuentra localizada en la diagonal árida- adopta, desde un punto de vista hidrológico, características de desierto con bajas precipitaciones (Bruniard 1982; Abraham de Vázquez et al. 2000; Mancini et al. 2005). Hacia el oeste existe una alta disponibilidad de agua y recursos asociados en sectores localizados, aunque se trata de espacios sometidos a una alta estacionalidad y de muy dificultosa o imposible ocupación bajo condiciones invernales (Barberena et al. 2015b; Bernal et al. 2017). Estas condiciones ecológicas podrían haber favorecido el establecimiento temprano de sistemas de movilidad y explotación estacional de áreas cordilleranas a partir de sectores bajos ubicados hacia el este, de manera tal que pudo utilizarse la heterogeneidad en la disponibilidad de recursos temporal y espacial a lo largo del ciclo anual. Sin embargo, establecer en qué medida la ocupación estacional del espacio se mantuvo a lo largo del Holoceno requiere obtener reconstrucciones paleoambientales precisas a escala regional, las cuales son escasas para algunas áreas de Neuquén, como el norte de la provincia.

En síntesis, los datos cronológicos y geográficos analizados en este trabajo permiten caracterizar el proceso de ocupación humana de Neuquén como temprano, mayormente continuo a lo largo del tiempo y espacialmente discontinuo. La profundidad temporal de los fechados radiocarbónicos disponibles alcanza ca. 12.000 años cal. AP, y la ocupación del espacio se incrementa significativamente hacia el Holoceno tardío. La distribución espacial de los sitios es discontinua y muestra concentraciones en áreas particulares cercanas a los cursos de agua permanente, mayoritariamente pericordilleranas, en tanto que en los espacios intermedios -las amplias planicies del este- no se registró una señal arqueológica marcada. La presentación de 12 nuevos fechados radiocarbónicos para la provincia del Neuquén contribuye a robustecer el patrón espacial descrito al incorporar datos de áreas y momentos para los que la información era aún escasa o nula. La evaluación de las causas del vacío de información existente para ciertas áreas geográficas -tanto si responde a sesgos o si se trata de una propiedad del registro arqueológico de la provincia- puede realizarse mediante la incorporación de sitios detectados para los cuales aún no se cuenta con fechados absolutos o respecto de los que solo se cuenta con fechados relativos. En este sentido, los resultados presentados aquí constituyen una guía para orientar el trabajo arqueológico futuro en la región.

\section{Agradecimientos}

Agradecemos a tres revisores anónimos, cuyos comentarios contribuyeron a mejorar la calidad de este trabajo. Los fechados radiocarbónicos originales presentados en este texto y los trabajos de campo de los que derivan fueron realizados con fondos provenientes del Gobierno de la Provincia del Neuquén, y de los subsidios otorgados por la Universidad Nacional de La Plata (PI 792), el Consejo Nacional de Investigaciones Científicas y Técnicas (PIP 0729) y la Agencia de Promoción Científica y Tecnológica (PICT 2134).

\section{REFERENCIAS CITADAS}

Abraham de Vázquez, E. M., K. Garleff, H. Liebricht, A. C. Regairaz, F. Schäbitz, F. A. Squeo, H. Stingl y C. Villagrán 2000 Geomorphology and Paleoecology of the Arid Diagonal in southern South America. Zeitschrift für Angewandte Geologie SH1: 55-61.

Barberena, R.

2013 Biogeografía, competencia y demarcación simbólica del espacio: modelo arqueológico para el norte de Neuquén. Intersecciones en Antropología 14: 367-381.

Barberena, R., L. Prates y M. E. De Porras 2015a The human occupation of northwestern Patagonia (Argentina): paleoecological and chronological trends. Quaternary International 356: 111-126. doi. org/10.1016/j.quaint.2014.09.055

Barberena, R., K. Borrazzo, A. Rughini, G. Romero, M. P. Pompei, C. Llano, M. E. de Porras, V. Durán, C. Stern, A. Re, D. Estrella, A. Forasiepi, F. Fernández, M. Chidiak, L. Acuña, A. Gasco y M. Quiroga

2015b Perspectivas arqueológicas para Patagonia septentrional: sitio Cueva Huenul 1 (provincia del Neuquén, Argentina). Magallania 43 (1): 137-163. dx.doi.org/10.4067/S0718-22442015000100009 
Barberena, R., G. Romero Villanueva, G. Lucero, M. V.

Fernández, A. A. Rughini y P. Sosa

2017 Espacios internodales en Patagonia septentrional: biogeografía, información y mecanismos sociales de interacción. Estudios Atacameños 56: 57-75. dx.doi. org/10.4067/S0718-10432017005000006

Bernal, V., V. A. Cobos, S. I. Perez y P. N. González 2017 La estructura espacial del registro bioarqueológico de la Provincia del Neuquén durante el Holoceno. En El poblamiento humano del norte del Neuquén. Estado actual del conocimiento y perspectivas, editado por $\mathrm{F}$. Gordón, R. Barberena y V. Bernal, pp. 123-144. Aspha, Buenos Aires.

Borrero, L.

2005 The archaeology of the Patagonian Deserts hunter-gatherers in a Cold Desert. En Desert Peoples. Archaeological Perspectives, pp. 142-157. Blackwell Publishing, Londres.

Bruniard, E.

1982 La diagonal árida Argentina: un límite climático real. Revista Geográfica 95: 5-20.

Crema, E. R., J. Habu, K. Kobayashi y M. Madella 2016 Summed probability distribution of ${ }^{14} \mathrm{C}$ dates suggests regional divergences in the population dynamics of the Jomon Period in Eastern Japan. PLOS ONE 11, e0154809. doi:10.1371/journal.pone.0154809

Crema, E. R., A. Bevan y S. Shennan

2017 Spatio-temporal approaches to archaeological radiocarbon dates. Journal of Archaeological Science 87: 1-9. doi:10.1016/j.jas.2017.09.007

Crivelli Montero, E., D. Curzio y M. Silveira 1993 La estratigrafía de la Cueva Traful I (Provincia del Neuquén). Præhistoria 1: 17-160.

Crivelli Montero, E., U. Pardiñas, M. Fernández, M.

Bogazzi, A. Chauvin, V. Fernández y M. Lezcano 1996 La Cueva Epullán Grande (Provincia del Neuquén, Argentina). Præhistoria 2: 17-160.

D’Elia, L., G. Páez, I. Hernando, I. Petrinovic, G. Villarosa, A. Bilmes, M. Bodaño, S. Guzmán, G. Borzi, S. Serra

Varela y C. Manzoni

2014 Erupciones históricas del volcán Tromen: análisis geomorfológico y geocronológico en su sector noroeste. Revista de la Asociación Geológica Argentina 71 (3): 444-448.

Della Negra, C. y P. Novellino 2005 Aquihuecó: un cementerio arqueológico en el norte de la Patagonia, valle del Curi Leuvú, Neuquén, Argentina. Magallania 33: 165-172. dx.doi.org/10.4067/ S0718-22442005000200011
Della Negra, C., P. Novellino, S. I. Perez, A. Hajduk y V. Bernal

2009 Investigaciones arqueológicas y bioarqueológicas en el sitio Aquihuecó (Norpatagonia): nuevos resultados. En Arqueología de Patagonia: una mirada desde el último confín, t. 2, editado por M. Salemme, F. Santiago, M. Álvarez, E. Piana, M. Vázquez y E. Mansur, pp. 669-676. Utopías, Ushuaia.

Della Negra, C., P. Novellino, M. Béguelin, F. Gordón, P. Gonzalez, R. Vázquez y V. Bernal

2014 Áreas de entierro en cazadores-recolectores del Noroeste de Patagonia: sitio Hermanos Lazcano (Chos Malal, Neuquén). Runa 35: 5-19.

Fernández, J.

1988-1990 La cueva de Haichol. Arqueología de los pinares cordilleranos del Neuquén. Anales de Arqueología y Etnología 43/45.

Gordón, F., M. Béguelin, P. Novellino y F. Archuby 2019 Inferencias paleodemográficas en el Noroeste de Patagonia a partir del sitio Aquihuecó, provincia del Neuquén, Argentina. Chungara. Revista de Antropología Chilena. En prensa.

Guichón, R., S. Muñoz y L. A. Borrero 2000 Datos para una tafonomía de restos óseos humanos en Bahía San Sebastián, Tierra del Fuego. Relaciones de la Sociedad Argentina de Antropología XXV: 297-311.

Hajduk, A. 1979 Datos arqueológicos Añelo Pehuenches. Informe inédito presentado ante el CONICET. MS.

Hajduk, A., P. Novellino, E. Cúneo, A. M. Albornoz, C. Della Negra y M. Lezcano

2007 Estado de avance de las investigaciones arqueológicas en el noroeste de la provincia del Neuquén (Departamentos Chos Malal y Minas, República Argentina) y su proyección futura. En Arqueología de Fuego-Patagonia. Levantando piedras, desenterrando huesos y... develando arcanos, editado por F. Morello, A. Prieto, M. Martinic y G. Bahamonde, pp. 467-477. Centro de Estudios del Cuaternario Fuego-Patagonia y Antártica (CEQUA), Punta Arenas.

Lema, V., C. Della Negra y V. Bernal 2012 Explotación de recursos vegetales silvestres y domesticados en Neuquén: implicancias del hallazgo de restos de maíz y algarrobo en artefactos de molienda del Holoceno tardío. Magallania 40 (1): 229 247. dx.doi.org/10.4067/S0718-22442012000100013 
Littleton, J.

2007 From the perspective of time: hunter gatherer burials in south-eastern Australia. Antiquity 81:10131081. doi.org/10.1017/S0003598X00096083

Littleton, J. y $\mathrm{H}$. Allen

2007 Hunter-gatherer burials and the creation of persistent places in southeastern Australia. Journal of Anthropological Archaeology 26: 283-298. doi. org/10.1016/j.jaa.2006.11.004

Mancini, M. V., M. M. Páez, A. R. Prieto, S. Stutz, M.

Tonello y I. Vilanova

2005 Mid-Holocene climatic variability reconstruction from pollen records $\left(32^{\circ}-52^{\circ} \mathrm{S}\right.$, Argentina). Quaternary International 132: 47-59. doi.org/10.1016/j. quaint.2004.07.013

Martin, F., R. Barberena y R. Guichón 2004 Erosión y huesos humanos. El caso de la localidad Chorrillos, Tierra del Fuego. Magallania 32: 125-142.

Perez, S. I., C. Della Negra, P. Novellino, P. N. González, V. Bernal, E. Cúneo y A. Hajduk

2009 Deformaciones artificiales del cráneo en cazadores-recolectores del Holoceno medio-tardío del Noroeste de Patagonia. Magallania 37(2): 77-90. dx.doi.org/10.4067/S0718-22442009000200005

Perez, S. I., P. N. González y V. Bernal

2016a Past population dynamics in Northwest Patagonia: an estimation using molecular and radiocarbon data. Journal of Archaeological Science 65: 154-160. doi. org/10.1016/j.jas.2015.11.013

Perez, S. I., M. B. Postillone, D. Rindel, D. Gobbo, P. N. Gonzalez y V. Bernal

2016b Peopling time, spatial occupation and demography of Late Pleistocene-Holocene human population from Patagonia. Quaternary International 425: 214-223. doi.org/10.1016/j.quaint.2016.05.004
Sanguinetti de Bórmida, A. C. y D. E. Curzio 1996 Excavaciones arqueológicas en el Sitio Piedra del Águila 11. Præhistoria 2: 43-100.

Shennan, S., S. S. Downey, A. Timpson, K. Edinborough, S. Colledge, T. Kerig, K. Manning y M. G. Thomas 2013 Regional population collapse followed initial agriculture booms in mid-Holocene Europe. Nat. Commun 4: 2486. doi.org/10.1038/ncomms3486

Stine, S.

1994 Extreme and persistent drought in California and Patagonia during mediaeval time. Nature 369: 546549. doi.org/10.1038/369546a0

Stuiver, M. y Reimer, P. J.

1993 Extended 14C data base and revised CALIB 3.0

$14 \mathrm{C}$ age calibration program. Radiocarbon 35 (1): 215230. doi.org/10.1017/S0033822200013904

Surovell, T. A., Finley, J. B., Smith, G. M., Brantingham, P. J. y R. Kelly 2009 Correcting temporal frequency distributions for taphonomic bias. Journal of archaeological Science 36 (8): 1715-1724. doi.org/10.1016/j.jas.2009.03.029

Timpson, A., S. Colledge, E. Crema, K. Edinborough, T. Kerig, K. Manning, M. G. Thomas y S. Shennan 2014 Reconstructing regional population fluctuations in the European Neolithic using radiocarbon dates: a new case-study using an improved method. Journal of Archaeological Science 52: 549-557. doi.org/10.1016/j. jas.2014.08.011

Torfing, $\mathrm{T}$.

2015 Neolithic population and summed probability distribution of 14C-dates. Journal of Archaeological Science 63: 193-198. doi.org/10.1016/j.jas.2015.06-004

\section{NOTAS}

1.- https://qgis.org/z 
Estructura espacial y dinámica temporal de la ocupación humana de Neuquén (Patagonia...Intersecciones en Antropología 20 (1), enero-julio. 2019. ISSN-e 1850-373X

\section{MATERIAL SUPLEMENTARIO}
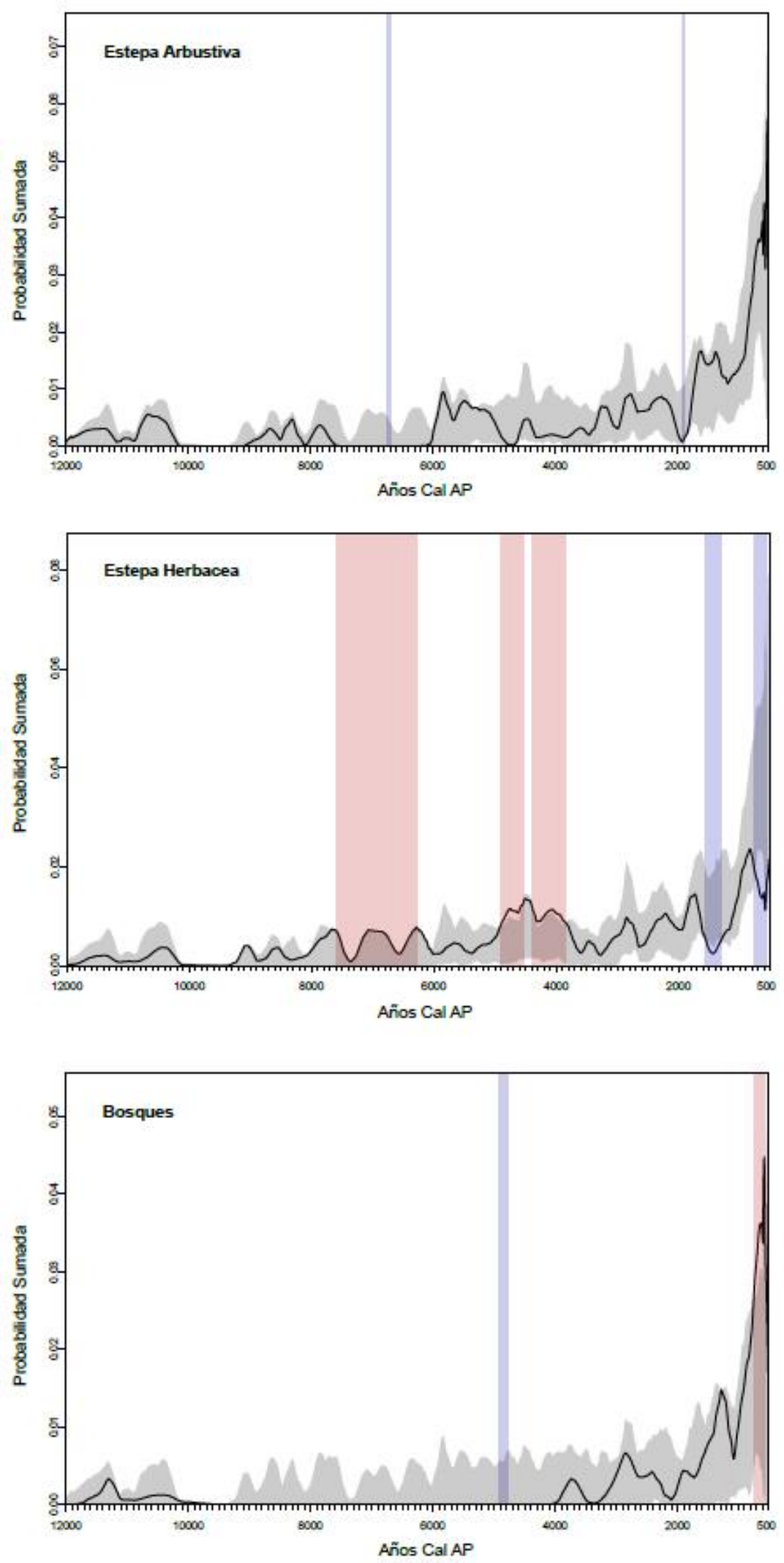
Estructura espacial y dinámica temporal de la ocupación humana de Neuquén (Patagonia...Intersecciones en Antropología 20 (1), enero-julio. 2019. ISSN-e 1850-373X

Figura 1 suplementaria. Resultado del análisis no-paramétrico de permutación por pares comparando la Distribución de Probabilidad Sumada de sitios de las tres áreas ecológicas consideradas por Barberena et al. (2015a): Bosques, Estepa Arbustiva y Estepa Herbácea. 
Tabla 1 suplementaria. Fechados radiocarbónicos estudiados en este trabajo. En la tabla se informa el fechado original, su desvío estándar y la edad calibrada. Se incluyen los tipos de sitio, agrupamientos geográficos y ecológicos utilizados en los análisis, así como la referencia bibliográfica original. Finalmente, se incluye la identificación única de cada fechado en la base de datos final, considerando los fechados de un mismo sitio que no difieren en más de 100 años entre ellos con un mismo nombre, e indicando que fechados fueron excluidos o promediados para generar la base de datos final de 142 fechados que fue utilizado en los análisis finales. 


\begin{tabular}{|c|c|c|c|c|}
\hline 1410 & Caso & Sitio & Edad Cal & \\
\hline & Aguada-de & Aguada-de & 769 & \\
\hline N2 & Alero-Cia & Alero-Cia & 943 & \\
\hline N3 & Alero-Cict & Alero-Cic & 1237 & \\
\hline N4 & Alero-del-[ & Alero-del-[ & 501 & \\
\hline N5 & ero-de-k & Alero-de-k & 1406 & \\
\hline N6 & Allero-de-k & Alero-de-k & 656 & \\
\hline N7 & Alero-de-k & Alero-de-k & 5084 & \\
\hline N144 & ero-l $\mid \mathrm{V}-\mathrm{d}$ & Alero-lV-d & 778 & \\
\hline N146 & $\operatorname{ero}-\mid V-d$ & Alero-lv-d & 924 & \\
\hline N142 & ero-lv-d & Alero-lv-d, & 2868 & \\
\hline N143 & ero-lv-d & Alero-lV-dי & 3792 & \\
\hline N147 & ero-ly-d & Alero-lW-d, & 4258 & \\
\hline N148 & ero-lv-d & Alero-lv-d & 5054 & \\
\hline N8 & Alero-Lariv & Alero-Larii & 678 & \\
\hline N9 & ero-Laris & Alero-Larii & 2836 & \\
\hline N10 & Alero-Las- & Alero-Las- & 560 & \\
\hline N11 & Alero-Los. & Alero-Los. & 729 & \\
\hline N12 & Alero-Los & Alero-Los. & 1374 & \\
\hline N13 & ero-Los. & Alero-Los. & 2983 & \\
\hline N14 & & & 3714 & \\
\hline N15 & Aquihuecc & Aquihuecc & 3919 & \\
\hline N16 & Aquihuecc & Aquihuece & 4151 & \\
\hline N17 & quihuecc & Aquihuecc & 4482 & \\
\hline N18 & ecc & Aquihuece & 4487 & \\
\hline N19 & & Aquih & 856 & \\
\hline N20 & Aquihuecc & Aquihuece & 4668 & \\
\hline N21 & puihs & Aquihuecc & 4698 & \\
\hline N22 & uihuecc & Aquihuece & 4750 & \\
\hline N23 & quiht & Aquihuecc & 4827 & \\
\hline N24 & & & & \\
\hline N25 & Arroyo-Cc & Arroyo-Cc & 11483 & \\
\hline N26 & Arroyo-Du & Arroyo-Du & 840 & \\
\hline N29 & Chorriaca- & Chorriaca & 1011 & \\
\hline N30 & Cochic & Cochico-N & 2445 & \\
\hline N31 & ollón-Ch & Collón-Ch & 253 & \\
\hline N33 & Cueva-del. & Cueva-del. & 522 & \\
\hline N34 & Cueva-del. & Cueva-del. & 1125 & \\
\hline N35 & (1) & Cueva-Hur & & \\
\hline N36 & & & & \\
\hline N37 & va-Hus & Cueva-Hü & & \\
\hline N38 & & Cueva-Hur & 1440 & \\
\hline & & & & \\
\hline
\end{tabular}

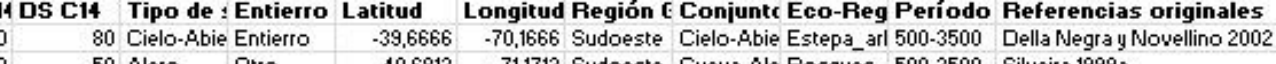
50 Alero Otro 55 Alero Otro 75 Alero Otro 50 Alero Otro 40 Alero Otro 120 Alero Dtro 120 Alero Otro 120 Alero Otro 70 Alero Otro 200 Alero Otro 130 Alero Otro 50 Alero Otro 80 Alero Otro 90 Alero Otro 90 Alero Otro 100 Alero Otro 80 Alero Otro 70 Cielo-Abie Entierro 66 Cielo-A.bie Entierro 61 Cielo-Abie Entierro 64 Cielo-Abie Entierro 55 Cielo-Abie Entierr 35 Cielo-Ábie Entierro 59 Cielo-Abie Entierro 59 Cielo-Abie Entierro 50 Cielo-Abie Entierr 96 Cueva Otro 33 Cielo-Abie Entierro 34 Cielo-Abie Entierro 90 Cielo-A.bie Otro 50 Cueva Otro 50 Cueva Otro 42 Cueva Otro 46 Cueva Otro 37 Cueva Otro 46 Cueva Otro \begin{tabular}{lll}
$-40,6812$ & $-71,173$ & Sudoeste Cueva-Ale Bosques $500-3500$ \\
\hline
\end{tabular}

$-38,999 \quad-68,4886$ Sudeste Cueva-Ale Estepa_arl 500-3500 Sanguinetti de Bórmida 1981 a b 38,999 -68,4886 Sudeste Cueva-Ale Estep_a arl 500-3500 Sanguinetti de Bórmida 1981 a-b $-39,2333 \quad-68,7667$ Sudeste Cueva-Ale Estepa_arl 500-3500 Borrero 1981 $\begin{array}{lll}-38,8605 & -70,444 & \text { Sudeste Cueva-Ale Estepa_arl 3501-6500 Borrero } 1981 \\ -38 & \end{array}$ \begin{tabular}{ll}
$-38,8605$ & $-70,444$ \\
\hline
\end{tabular} $-38,8605 \quad-70,444$ Sudoeste Cueva-Ale Estepa he 500-3500 Perrotay Pereda 1987

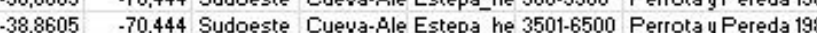
$-38,8605 \quad-70,444$ Sudoeste Cueva-Ale Estepa he 3501-6500 Perrota y Pereda 1987 $-38,8605 \quad-70,444$ Sudoeste Cueva-Ale Estepa_he 3501-6500 Perrota y Pereda 1987 $-40,6021 \quad-71,2283$ Sudoeste Cueva-Ale Bosques $500-3500$ Silveira 19996 $-40,6021 \quad-71,2283$ Sudoeste Cueva-Ale Bosques $500-3500$ Silveira 1999b $-40,6037 \quad-71,3584$ Sudoeste Cueva-Ale Bosques $500-3500$ Silveira 1987, Silveira et al. 2014 $-40,6431 \quad-71,3187$ Sudoeste Cueva-Ale Bosques $500-3500$ Silveira 1996 $-40,6431-71,3187$ Sudoeste Cueva-Ale Bosques $500-3500$ Silveira 1996 $-40,6431 \quad .71,3187$ Sudoeste Cueva-8le Bosques $3501-6500$ Silueira 1996

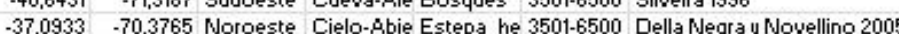
$-37,0933 \quad-70,3765$ Noroeste Cielo-Abie Estepa_he 3501-6500 Perez et al. 2009 $-37,0933 \quad-70,3765$ Noroeste Cielo-Abie Estepa_he 3501-6500 este estudio $-37,0933 \quad-70,3765$ Noroeste Cielo-Abie Estepa he 3501-6500 Perez et al. 2009 $-37,0933 \quad-70,3765$ Noroeste Cielo-Abie Estepa_he $3501-6500$ este estudio

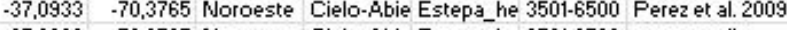
$-37,0933 \quad-70,3765$ Noroeste Cielo-Abie Estepa_he 3501-6500 este estudio $-37,0933 \quad-70,3765$ Noroeste Cielo-Abie Estepa_he 3501-6500 este estudio $-37,0933 \quad-70,3765$ Noroeste Cielo-46ie Estepa_he 3501-6500 este estudio $-47,0933$ - 70,3765 Noroeste Clelo-Abie Estepa_he 6501-9500 este estudio

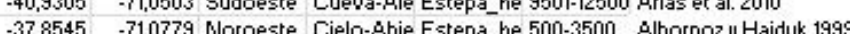

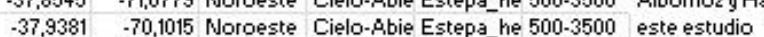
$\begin{aligned}-36,4841 & -70,2213 \\ -30 & \text { Noroeste Cielo-Abie Estepa_he 500-3500 este estudio }\end{aligned}$ $-30,0702 \quad-68,1069$ Sudeste Cielo-Abie Estepa_arl 500-350 Albero y, Angiolini 1900 $-40,0702 \quad-70,0046$ Sudoeste Cueva-Ale Estepa_arl 500-3500 Barberena et al. 2002 $-36,9587 \quad-69,8237$ Noroeste Cueva-Ale Estepa_arl 500-3500 Barberena et al. 2015 $-36,9587 \quad-69,8237$ Noroeste Cueva-Ale Estepa_arl 500-3500 Barberena et al. 2015 $-36,9587-69,8237$ Noroeste Cueva-Ale Estepa_arl 500-3500 Barberena et al. 2010

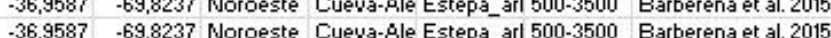

Identific: Fechados Excluidos o Promediados (x) A.guada-del-Overo_1 Alero-Cicuta_ Alero-del-Dique_1 Alero-de-los-Alamos_-1 Alero-de-los-Sauces Alero-lv-de-Tromen-1 Alero-lV-de-Tromen-2 Alero-lV-de-Tromen-3 Alero-lV-de-Tromen-4 Alero-lV-de-Tromen-5 Alero-V-de-Tromen_Alero-Lariviere-1 1 Alero-Las-Mellizas Alero-Los-Cipreses_Alero-Los-Cipreses_2 Alero-Los-CipresesAlero-Los-Ciprese 1 Aquihueco-1

Aquihuer $x$

Aquihuer:

Aquihuer:

Aquihuer:

Aquihuer:

Aquihuer:

Aquihuer:

Aquihueco_6 6 Arroyo-Corral-2_1 Arroyo-Dulce Cochico 1 Collón-Chico_1 Cueva-del-Choique 1 Cueva-del-Choique_2 Cueva-Huenul_1 Cueva-Huenul_Cueva-Huenul_3 Cueva-Huenul_4
Cueva-Huenul_5 


\begin{tabular}{|c|c|}
\hline $\mathrm{N} 43$ & Cueva-Hur Cueva-Hut \\
\hline N44 & Cueva-Hu Cueva-Hur \\
\hline N45 & Cueva-Hur Cueva-Hut \\
\hline N46 & Cueva-Hur Cueva-Hu \\
\hline N48 & Cueva-Laç Cueva-Lạ \\
\hline N47 & Cueva-Lac Cueva-Lac \\
\hline N49 & Cueva-Pai Cueva-Pai \\
\hline N50 & Cueva-Pat Cueva-Pal \\
\hline N51 & Cueva-Pal Cueva-Pal \\
\hline N52 & Cueva-Pal Cueva-Pa \\
\hline N53 & Cueva-Pal Cueva-Pal \\
\hline N55 & Cueva-Rul Cueva-Rul \\
\hline N56 & Cueva-Tra Cueva-Tra \\
\hline N57 & Cueva-Tra Cueva-Tra \\
\hline N58 & Cueva-Tra Cueva-Tra \\
\hline N59 & Cueva-Tra Cueva-Tra \\
\hline N60 & Cueva-Tra Cueva-Tra \\
\hline N61 & Cueva-Tra Cueva-Tra \\
\hline N62 & Cueva-Tra Cueva-Tra \\
\hline N63 & Cueva-Tra Cueva-Tra \\
\hline N64 & Cueva-Tra Cueva-Tra \\
\hline N65 & Cuyin-Mar Cuyin-Mar \\
\hline N66 & Cugin-Mar Cuyin-Mar \\
\hline N67 & Epullan-Cr Epullan-Cr \\
\hline N68 & Epullan-Cr Epullan-Cr \\
\hline N70 & Epullan-Gr Epullan-Gr \\
\hline N71 & Epullan-Gir Epullan-Gir \\
\hline N72 & Epullan-Gr Epullan-Gir \\
\hline N73 & Epullan-Gir Epullan-Gir \\
\hline N74 & Epullan-Gr Epullan-Gir \\
\hline N75 & Epullan-Gr Epullan-Gir \\
\hline N76 & Epullan-Gr Epullan-Gir \\
\hline N77 & Epullan-Gir Epullan-Gir \\
\hline N78 & Epullan-Gir Epullan-Gir \\
\hline N79 & Epullan-Gr Epullan-Gir \\
\hline N80 & Epullan-Gr Epullan-Gir \\
\hline N81 & Epullan-Gr Epullan-Gir \\
\hline N82 & Gubevi-1 Gubevi \\
\hline M18 & Haichol-6 Haichol \\
\hline & \\
\hline
\end{tabular}

91 Cueva Otro 60 Cueva Otro 39 Cueva Otro 98 Cueva Otro 95 Cueva Otro 100 Cueva Otro 60 Cueva Otro 60 Cueva Otro 60 Cueva Otro

60 Cueva Otro

$\begin{array}{ll}70 \text { Cueva } & \text { Otro } \\ 37 \text { Cueva } & \text { Otro }\end{array}$

37 Cueva Otro

40 Cueva Otro

115 Cueva Otro

60 Cueva Otro 250 Cueva Otro 285 Cueva Otro 105 Cueva Otro 105 Cueva Otro 230 Cueva Otro 240 Cueva Otro 85 Cueva Otro 50 Cueva Otro 50 Cueva Otro 50 Cueva Dtro Oro 50 Cueva Dtro

50 Cueva Otro

50 Cueva Otro

50 Cueva Otro

70 Cueva Drro

Oro

50 Cueva atro

70 Cueva Otro

100 Cueva Otro

43 Cielo-Abie Entierro

50 Cueva Orie
-36,9587 -69,8237 Noroeste Cueva-Ale Estepa_arl 9501-12500 Barberena et al. 2015

-36,9587 -69,8237 Noroeste Cueva-Ale Estepa_arl 9501-12500 Barberena et al. 2015

$-36,9587 \quad-69,8237$ Noroeste Cueva-Ale Estepa_arl 9501-12500 Barberena et al. 2010

$\begin{array}{llll}40,7011 & -712774 & \text { Noroeste Cueva-Ale Estepa_arl 9501-12500 Barberena et al. } 2015\end{array}$

\begin{tabular}{llll}
$-40,7011$ & $-71,2774$ & Sudoeste Cueva-Ale Bosques $500-3500$ & Silveira et al 2013 \\
\hline
\end{tabular}

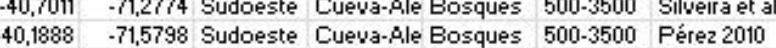

$-40,1888 \quad-71,5798$ Sudoeste Cueva-Ale Bosques $500-3500$ Perez 2010

$-40,1888$

$-40,1888$

$-40,1888$

$-37,7568$
$-40,7304$

$-40,7304$

$-40,7304$

$-40,7304$

$-40,7304$

$-40,7304$

$-40,7304$

$-40,7304$

$-40,7304$

$-40,7631$
$-40,7631$
$-40,3904$

$-40,3904$

$-40,3904$

$-40,3904$

$-40,3904$

$-40,3904$

$-40,3904$

$-40,3904$

$-40,3904$

$-40,3904$

$-40,3904$

$-40,3904$

$-37,1773$

$-38,5858$

$-38,5858$

71,5798 Sudoeste Cueva-Ale Bosques 500-3500 Ṕ 2010

71,5798 Sudoeste Cueva-Ale Bosques 500-3500 Pérez 2010

71,5758 Sudoeste Cueva-Ale Bosques 500-3500 Perez 2010

.71, Cueva-Q.le Estepa_arl 3501-6500 este estudio

7, Sudoeste Cueva-Ale Estepa_he 500-3500 Crivelli Montero et al. 1993

71,13 Sudoeste Cueva-Ale Estepa_he 500-3500 Crivelli Montero et al. 1993

-71,13 Sudoeste Cueva-Ale Estepa-he 6501-9500 Crivelli Monteroet al. 1993

71,13 sudoeste Cueva-Ale Estepa_he 6501-9500 Crivelli Montero et al. 1993

$-71,13$ sudoeste Cueva-gle Estepa_he 6501-9500 Crivelli Monteroet al. 1993

$-71,13$ Sudoeste Cueva-Ale Estepa_he 6501-9500 Crivelli Montero et al. 1993

7113 sudoeste Cueva-Ale Estepa_he 9501-12500 Crivelli Montero et al. 1993

$-71,1873$ Sudoeste Cueva-Ale Bosques 9501-12500 Ceballos 1982

$-71,1873$ Sudoeste Cueva-Ale Bosques 9501-12500 Ceballos 1982
$-70,1841$ Sudoeste Cueva-Ale Estepa_arl 500-3500 Crivelli Montero et al. 1996

$-70,1841$ Sudoeste Cueva-Ale Estepa_arl 500-3500 Crivelli Montero et al. 1996

-70,1841 Sudoeste Cueva-Ale Estepa_arl 500-3500 Crvelli Monteroet al. 1936

701841 Sudeste Cueva Ale Estopa 15003500 Civeli Montes a 1936

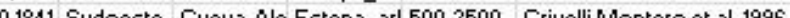

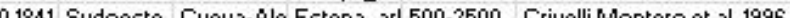

701841 Sudoeste Cuevo Ale Esteparl 500-3500 Grivelli Monteroet a 1996

70.1841 Sudoeste Cueva-8le Estepa $500-3500$ Crivelli Montero a 1996

70.1841 Sudoeste Cuevo Ale Estepa 3501-6500 Crivelli Monteroet a 1996

70,1841 Sudoeste Cuevo Ale Estepa $6501-9500$ Crivelli Montero et al 1996

70,1841 Sudoeste Cueua-Ale Estepa arl 6501-9500 Crivelli Monteroet al 1996

$-70,1841$ Sudoeste Cueva-Ale Estepa-arl 6501-9500 Crivelli Montero et al 1996

$-70,1841$ Sudoeste Cueua-Ale Estepa arl 9501-12500 Crivelli Monteroet al 1996

70,6697 Noroeste Cielo-A.bie Estepa_he 500-3500 Della Negra 2008

70,6714 Noroeste Cueva-Ale Estepa_he 500-3500 Fernández 1988-1990

\section{Cueva-H}

Cueva-H :

Cueva-Huenul_ 9

Cueva-Huenul_10

Cueva-Lagartiia_ 1

Cueva-Parque-Diana_

Cueva-Parque-Diana_2

Cueva-P :

Cueva-Parque-Diana_4

Cueva-Riuka_1

Cueva-Traful_1

Cueva-Traful_2

Cueva-Traful 3

Cueva-Traful-4

Cueva-Traful 5

Cueva-Traful_6

Cueva-Traful-7

Cueva-Traful_8

Cueva-Traful_9

Cuyin-Manzano-1

Epullan-1 x

Epullan-1:

Epullan-Grande-1

Epulan-Grande

Epullan-1 :

Epullan-1 2

Epulangrande-4

Epulan-Grande 6

Epullan-Grande?

Epullan-Grande-

Epullan-Giande-

Epullan-Grande-10

Epullan-Grande-11

Gubevi-1

Haichol_1

Haichol_x 


\begin{tabular}{|c|c|}
\hline N92 & Haichol-10 Haichol \\
\hline N93 & Haichol-11 Haichol \\
\hline N94 & Haichol-12 Haichol \\
\hline N95 & Haichol-13 Haichol \\
\hline N96 & Haichol-14 Haichol \\
\hline N97 & Haichol-15 Haichol \\
\hline N98 & Haichol-16 Haichol \\
\hline N100 & Haichol-18 Haichol \\
\hline N101 & Haichol-19 Haichol \\
\hline N99 & Haichol-17 Haichol \\
\hline N102 & Haichol-2C Haichol \\
\hline N103 & Haichol-21 Haichol \\
\hline N104 & Haichol-22 Haichol \\
\hline N105 & Haichol-2: Haichol \\
\hline N106 & Haichol-24 Haichol \\
\hline N107 & Haichol-2E Haichol \\
\hline N108 & Haichol-2E Haichol \\
\hline N109 & Haichol-2ì Haichol \\
\hline N110 & Haichol-2\& Haichol \\
\hline N111 & Haichol-2؟ Haichol \\
\hline N112 & Haichol-3C Haichol \\
\hline N113 & Haichol-31 Haichol \\
\hline N114 & Haichol-32 Haichol \\
\hline N115 & Haichol-3: Haichol \\
\hline N116 & Haichol-34 Haichol \\
\hline N117 & Haichol-3E Haichol \\
\hline N118 & Haichol-3E Haichol \\
\hline N119 & Haichol-3ì Haichol \\
\hline N120 & Haichol-3\& Haichol \\
\hline N121 & Haichol-3\& Haichol \\
\hline N122 & Haichol-4C Haichol \\
\hline N123 & Haichol-41 Haichol \\
\hline N124 & Haichol-4z Haichol \\
\hline N125 & Haichol-4: Haichol \\
\hline N126 & Haichol-44 Haichol \\
\hline N127 & Hermanos Hermano \\
\hline N128 & Hermanos Hermano \\
\hline N129 & Lago-Melii Lago-Mel \\
\hline N130 & Lago-Meli Lago-Mel \\
\hline N131 & Lago-Meli Lago-Mel \\
\hline N132 & Laguna-Mi Laguna-M \\
\hline N133 & Laguna-Mi Laguna-M \\
\hline
\end{tabular}

\begin{tabular}{|c|c|c|c|c|}
\hline & Cueva & Otro & $-38,5858$ & $.70,6714$ \\
\hline & Cueva & Otro & $-38,5858$ & $-70,6714$ \\
\hline & Cueva & Otro & $-38,5858$ & $.70,6714$ \\
\hline 70 & Cueva & Otro & $-38,5858$ & $.70,6714$ \\
\hline 00 & Cueva & Otro & $-38,5858$ & $.70,6714$ \\
\hline & Cueva & Otro & $-38,5858$ & $\cdot 70,6714$ \\
\hline & Cueva & Otro & $-38,5858$ & $.70,6714$ \\
\hline & Cueva & Otro & $-38,5858$ & $.70,6714$ \\
\hline & Cueva & Entierro & $-38,5858$ & $.70,6714$ \\
\hline 110 & Cueva & Otro & $-38,5858$ & $.70,6714$ \\
\hline 00 & Cueva & Otro & $-38,5858$ & $.70,6714$ \\
\hline & Cueva & Otro & $-38,5858$ & $.70,6714$ \\
\hline & Cueva & itro & $-38,5858$ & $.70,6714$ \\
\hline & Cue & & 58 & \\
\hline 00 & Cueva & Otro & $-38,58$ & $.70,6714$ \\
\hline 00 & Cueva & Otro & $-38,58$ & $-70,6714$ \\
\hline 00 & Cueva & Otro & $-38,5858$ & $.70,6714$ \\
\hline & Cueva & Entierr & $-38,5858$ & $.70,6714$ \\
\hline & Cue & & & \\
\hline & Cueva & 0 & & \\
\hline 70 & Cueva & Entierro & $-38,5858$ & $-70,6714$ \\
\hline 86 & Cueva & Otro & $-38,5858$ & $-70,6714$ \\
\hline 115 & Cueva & & 8.5858 & \\
\hline & Cue & & & \\
\hline 00 & Cueva & & & \\
\hline 100 & Cueva & Otro & $-38,5858$ & $.70,6714$ \\
\hline 130 & Cueva & Entierr & 8,5858 & $.70,6714$ \\
\hline & & & & \\
\hline 60 & Cue & Entier & 58 & \\
\hline 110 & Cue & & & \\
\hline 70 & Cueva & Entierro & 8.5858 & $.70,6714$ \\
\hline & Cue: & & & \\
\hline 130 & & & $-38,5$ & $.70,6$ \\
\hline 75 & Cueva & Otro & $-38,5858$ & $.70,6$ \\
\hline 120 & Cueva & Otr & $-38,5858$ & \\
\hline & Cielo-A & & 5 & \\
\hline 33 & Cielo-A & Entierro & & $.70,4$ \\
\hline 80 & Cielo-Abie & Otro & $-40,33$ & $.71,3169$ \\
\hline 60 & Cielo-Abie & Otro & $-40,3325$ & $.71,3169$ \\
\hline & & & & \\
\hline & & & 84 & \\
\hline & & & & \\
\hline & & & & \\
\hline
\end{tabular}

Cueva-Ale Estepa_he 500-3500 Fernández 1988-1990 Cueva-Rle Estepa_he 500-3500 Fernández 1988-1990 Cueva-Ale Estepa_he 500-3500 Fernández 1988-1990 Cuevale Estepa-he 500-3500 Fernandez 1988-1990 Cuevoris Estepa- 500-3500 Fernandez 1988-1930 Cueva-Ale Estea he 500-3500 Fenndez $1980-1990$ Cuevale Estepa 500-3500 Ferníndez 1980-1990 Cueva-Ale Estepa_he 500-3500 Fernandez y Panarello 200 Cueva-Ale Estepa_he 500-3500 Fernández 1988-1990 Cueva-Ale Estepa_he 500-3500 Fernández 1988-1990 Cueva-Ale Estepa_he 500-3500 Fernández 1988-1990 Cueva-Ale Estepa_he 500-3500 Fernández 1988-1990 Cueva-Ale Estepa_he 500-3500 Fernández 1988-1990 Cueva-Ale Estepa_he 500-3500 Fernández $1988-1990$
Cueva-Ale Estepa_he 500-3500 Fernandez y Panarello 200 Cueva-Ale Estepa_he 500-3500 Fernandez y Panarello
Cueva-Ale Estepa_he 3501-6500 Fernández 1988-1990
Cueva-Ale Estepa_he 3501-6500 Fernández 1988-1990 Cueva-Ale Estepa_he 3501-6500 Fernandez y Panarello 2001 Cueva-Ale Estepa_he 3501-6500 Fernández 1988-1990 Cueva-Ale Estepa_he 3501-6500 Fernández 1988-1990 Cueva-Ale Estepa_he 3501-6500 Fernández 1988-1990 Cueva-Ale Estepa he 3501-6500 Fernández 1988-1990 Cueva-Ale Estepa_he 3501-6500 Fernández 1988-1990 Cueva-Ale Estepa_he 3501-6500 Fernandez y Panarello 2001 Cueva-Ale Estepa_he 3501-6500 Fernandez y Panarello 2001 Cueva-Ale Estepa_he 3501-6500 Fernández 1988-1990 Cueva-Ale Estepa_he 3501-6500 Fernandez y Panarello 2001 Cueva-Ale Estepa_he 6501-9500 Fernández 1988-1990 Cueva-Ale Estepa_he 6501-9500 Fernández 1988-1990 Cueva-Ale Estepa_he 6501-9500 Fernández 1988-1990 Cueva-Ale Estepa_he 6501-9500 Fernández 1988-1990 Cielo-Abie Estepa he 3501-6500 Hajduk et al 2000 Cielo-Abie Estepa_he 3501-6500 este estudio $\begin{array}{lll}\text { Cielo-Abie Bosques } & 500-3500 & \text { Pérez } 2010 \\ \text { Cielo-Abie Bosques } & 500-3500 & \text { Pérez } 2010\end{array}$ $\begin{array}{lll}\text { Cielo-A.bie Bosques } & 500-3500 & \text { Pérez } 2010 \\ \text { Cielo-Abie Bosques } & 500-3500 & \text { Pérez } 2010\end{array}$ $\begin{array}{lll}\text { Cielo-Abie Bosques } & 500-3500 & \text { Pérez } 2010 \\ \text { Cielo-Abie Estepa_he 500-3500 Stern et al. } 2012\end{array}$ Cielo-Abie Estepa_he 500-3500 Stern et al. 2012

Cueva-Âle Bosques 500-3500 Silveira 1995 Cueva-Ale Estepa_he 500-3500 Fernández 1988-1990

Haichol_a Haichol_:

Haichol_:

Haichol_ $x$

Haichol_:

Haichol_:

Haichol_6

Haichol_:
Haichol_
Hachos

Haichol_:

Haichol- 2

Haichol_ $x$

Haichol_:

Haichol_:

Haichol_ :

Haichol_:

Haichol_ 2

Haichol_ta

Haichol_:
Haichol_:

Haichol__ $x$

Haichol :

Haichol_ x

Haichol_14

Haichol_15

Haichol_:

Haich

Haichol

Haichol 19

Haichol 20

Haichol_21

Haichol_22

Hermanos-Lazcano_

Lago-Me $\mathbf{x}$

Lago-Me $x$

Lago-Meliquina_ 2

Laguna-Monticulo-1_- Montículo-1_2 


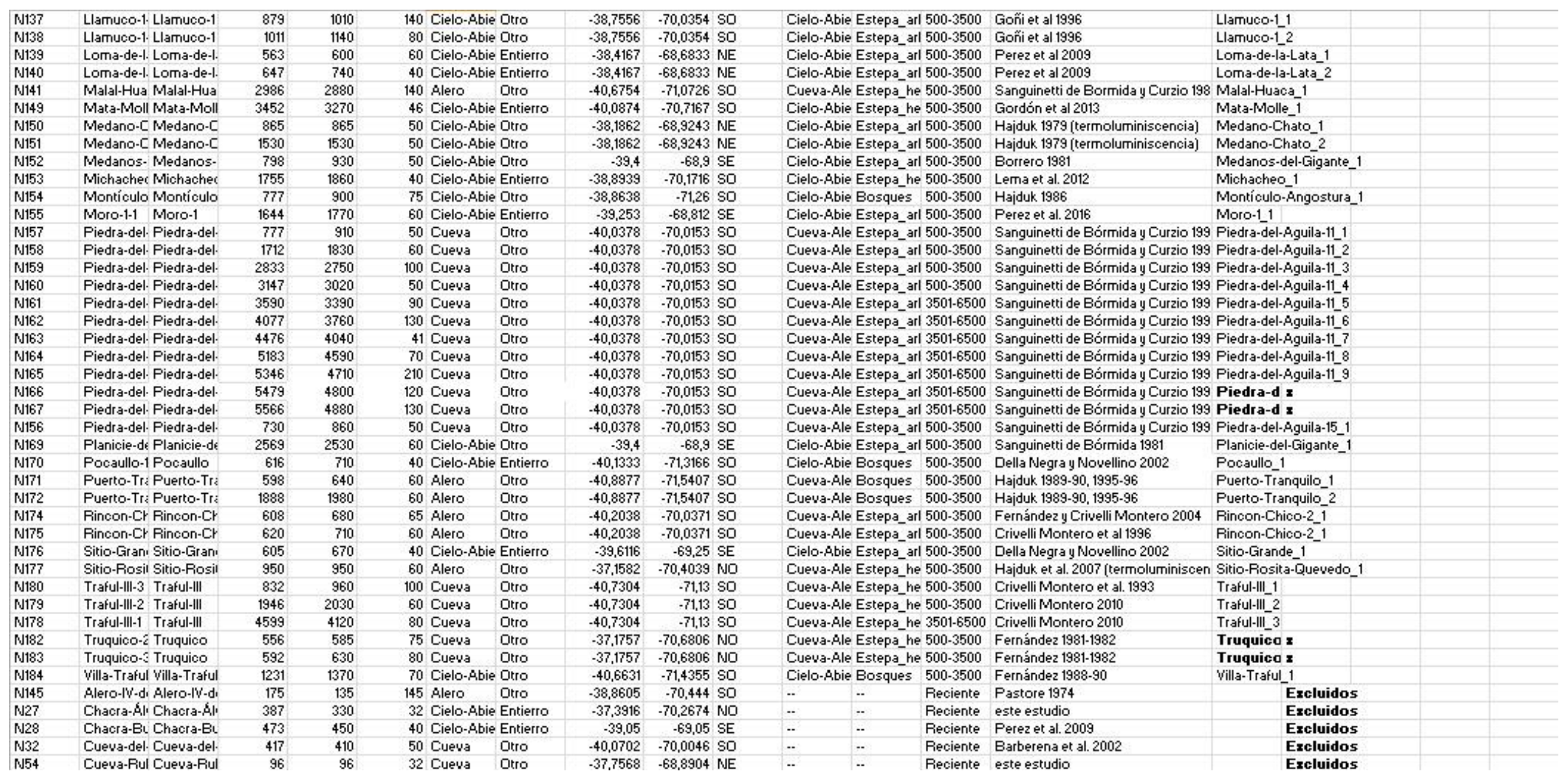




\begin{tabular}{|c|c|c|c|c|c|c|c|c|c|}
\hline N69 & Epullan-Gir & Epullan-Gir & 367 & 320 & 60 & Cueva & Otro & $-40,3904$ & $.70,1841$ \\
\hline N83 & Haichol-1 & Haichol & 197 & 225 & 85 & Cueva & Otro & $-38,5858$ & $.70,6714$ \\
\hline N84 & Haichol-2 & Haichol & 353 & 350 & 120 & Cueva & Otro & $-38,5858$ & $.70,6714$ \\
\hline N85 & Haichol-3 & Haichol & 394 & 365 & 45 & Cueva & Otro & $-38,5858$ & $.70,6714$ \\
\hline N86 & Haichol-4 & Haichol & 408 & 420 & 110 & Cueva & Otro & $-38,5858$ & $.70,6714$ \\
\hline N87 & Haichol-5 & Haichol & 454 & 470 & 110 & Cueva & Otro & $-38,5858$ & $.70,6714$ \\
\hline N168 & Piera-1 & Piera & 197 & 229 & 32 & Cielo-Abie & Entierro & $-38,7033$ & $-69,9832$ \\
\hline N173 & Retamal-1- & - Retamal 1 & 164 & 190 & 60 & Cielo-Abie & Entierro & $-38,983333$ & $-68,7167$ \\
\hline N181 & Truquico-1 & Truquico & 383 & 350 & 70 & Cueva & Otro & $-37,1757$ & $.70,6806$ \\
\hline
\end{tabular}

Reciente Crivelli Montero et al. 1996

Rieciente Fernández 1988-1990

Reciente Fernández 1988-1990

Rieciente Fernández 1988-1990

Reciente Fernández 1988-1990

Reciente Fernández 1988-1990

Reciente este estudio

Reciente Perez et al. 2009

Reciente Fernández 1981-1982
Excluidos

Excluidos

Excluidos

Excluidos

Excluidos

Excluidos

Excluidos

Excluidos

Excluidos 